Sharif University of Technology
Scientia Iranica
SCIENTIA
I RAN I CA
http://scientiairanica.sharif.edu

\title{
Smart-home electrical energy scheduling system using multi-objective antlion optimizer and evidential reasoning
}

\author{
A. Kaveh* and Y. Vazirinia \\ Centre of Excellence for Fundamental Studies in Structural Engineering, Iran University of Science and Technology, Narmak, \\ Tehran, Postal Code 16846-13114, Iran.
}

Received 14 June 2019; received in revised form 23 July 2019; accepted 10 August 2019

\author{
KEYWORDS \\ Building energy \\ management system; \\ Multi-objective \\ antlion optimizer; \\ Demand side \\ scheduling; \\ Multi-criteria decision \\ making; \\ $\mathrm{CO}_{2}$ emission; \\ Evidential reasoning.
}

\begin{abstract}
Smart-Home Energy Management Systems (SHEMSs) are widely used for energy management in smart buildings. Energy management in smart homes is an arduous task and necessitates efficient scheduling of appliances in buildings. Scheduling of smart appliances is usually enmeshed by various and sometimes contradictory criteria, which should be considered concurrently in the scheduling process. Multi-Criteria Decision Making (MCDM) techniques are able to select the most suitable alternative among copious ones. This paper tailors a comprehensive framework which merges MCDM techniques with Evolutionary Multi-Objective Optimization (EMOO) techniques for selecting the most proper schedule for appliances by creating a trade-off between optimization criteria. A Multi-Objective Ant Lion Optimizer (MOALO) was tailored and tested on a smart home case study to detect all the Pareto solutions. A benchmark instance of the appliance scheduling was solved employing the proposed methodology. Then, Shannon's entropy technique was employed to find the weights corresponding to the objectives. Finally, the acquired Pareto optimal solutions were ranked utilizing the Evidential Reasoning (ER) method. By inspecting the efficiency of every solution considering multiple criteria such as unsafety, electricity cost, delay, Peak to Average Ratio (PAR), and $\mathrm{CO}_{2}$ emission, effectiveness of the proposed approach in enhancing the method for smart appliance scheduling was confirmed.
\end{abstract}

(C) 2020 Sharif University of Technology. All rights reserved.

\section{Introduction}

During the previous decade, the electrical energy consumption of residential sectors has increased rapidly all around the world due to the expansion in appliance

\footnotetext{
*. Corresponding author. Tel.: +982144249493; Fax: +9821 7r7240398

E-mail addresses: alikaveh@iust.ac.ir (A. Kaveh) yasinvazirinia@gmail.com (Y.Vazirinia)
}

doi: $10.24200 /$ sci. 2019.53783 .3412 ownership [1]. Therefore, improvement in the energy efficiency of electrical facilities is very influential for energy-saving in buildings, reducing the loads on electrical grids, and decreasing the carbon footprint. Consequently, electricity conservation in buildings not only results in saving fossil fuels but also prevents capacity expansion in the power sector [2,3]. Many research results are available for supporting the decisions in the management of networks $[4,5]$. The emergence of smart homes and the Internet has led to an opportunity for automatic operation, scheduling of the appliances, and energy management in residential buildings. 
In recent years, utilization of metaheuristic optimization algorithms to overcome real-life problems has turned out to be a topic of huge interest for related studies. The main objective of such techniques is to sufficiently explore the search space to achieve global or quasi-global solutions. These algorithms have received increasing attention of both academia and industry [6,7]. Many metaheuristics with various philosophies and characteristics are developed and implemented in an immense domain of fields with variations of single-objective optimization, e.g. Genetic Algorithm (GA) [8], Particle Swarm Optimization (PSO) [9], colliding bodies optimization [10], Vibrating Particles System (VPS) [11], Ant Lion Optimizer (ALO) [12], etc., and Multi-Objective Optimization (MOO), e.g. non-dominated sorting GA [13], multiobjective PSO [14,15], multi-objective VPS [16], MultiObjective Ant Lion Optimizer (MOALO) [17], etc. Some applications of metaheuristics to engineering optimization problems can be found in $[18,19]$. A newly developed MOALO, which mimics the trapping mechanism of antlions in nature, is selected in this study [17]. Like many metaheuristic algorithms, MOALO has the advantage of simplicity and flexibility.

Electric energy management is involved in various problems in which the decision-maker needs to circumscribe possible scheduling options and select the one with optimal solutions, which have balance among different objectives. The Evolutionary Multi-Objective Optimization (EMOO) methods represent a suitable and practical procedure that supports robust and concurrent optimization of contradictory and frequently incommensurable objectives. In a real system, it is unwise to reach a decision that is founded on meeting only one criterion during the process of decisionmaking. This proves the exigency of using multiplecriteria assessment techniques to obtain a solution that meets all the decision-making presumptions with an agreeable degree of satisfaction [20].

Diverse EMOO procedures have been utilized to solve Multi-Objective Home Appliance Scheduling Problems (MOHASPs) and their sequent optimal Pareto solutions have been produced, plotted, and widely announced. Nevertheless, no effort has been put into selecting a solution that meets the objectives within a reasonable level. Due to the intimately interwoven multi-criteria nature of scheduling problems, ascertaining which solution is the best alternative can be challenging [21]. These criteria, also called objectives, are frequently incompatible. Multi-Criteria Decision Making (MCDM) approaches offer an efficient mechanism for selecting a suitable Pareto member [22]. In this research, an MCDM approach is combined with an EMOO technique to utilize the capability of the optimization technique for finding the optimal Pareto front (alternatives) and the capability of the
MCDM for simultaneously ranking them by various criteria to produce a single-compromise solution with a computational background.

Since the end of the 1970 s, several MCDM methods have been proposed to assist decision-makers in finding values of the criteria and the alternatives based on their preferences [23]. The goal of using MCDM techniques in decision making is to ease the process of organizing and harmonizing the requisite data in evaluation so that users feel comfortable with and sure in making decisions [24]. However, MCDM techniques are dissimilar in terms of theoretical background, formulation, questions, and types of input and/or output [25]. They can be divided into three main categories [26]: (a) value measurement techniques; (b) goal, aspiration, and reference level techniques; and (c) outranking techniques.

In the value measuring technique, a numerical value is assigned to every alternative, which intimates the rank of a solution versus others. Then, for making a trade-off between multiple criteria, each criterion is weighted based on decision-maker-accepted criteria [21]. The Analytical Hierarchy Process (AHP) [27] and multi-attribute utility theory fall into this category. The second category involves iterative methods that indicate closeness of the solutions to a predefined goal or reference level. The examples of this category are Evidential Reasoning (ER) [28] and the Technique for Order of Performance by Similarity to Ideal Solution (TOPSIS) [29]. Generally, these techniques are focused on purifying the most inappropriate alternatives at the initial stage of the multi-criteria assessment process [24]. In the outranking techniques, the alternatives are sorted by a pairwise matching and if enough proof exists to show that alternative (a) is preferable to alternative (b), it is assumed that alternative (b) is outranked by (a). PROMETHEE [30] and ELECTRE [31] are examples of the outranking techniques.

Regarding the number of MCDM techniques available, the decision on selecting a suitable decision support tool can be challenging to justify. None of the techniques are perfect nor can they be applied to all problems. Every technique has its limitations, particularities, hypotheses, premises, and perspectives. There are different ways of selecting a suitable MCDM technique to solve particular problems. One way is to consider the requisite input information, that is, the data and parameters of the method and, consequently, the modeling effort, as well as to take into account the outcomes and their granularity [32].

In this research, the ER technique is accommodated to produce an efficient Pareto solutions ranking system and discover the most appropriate solution. ER technique considers incomplete assessments or ignorance as a kind of probabilistic uncertainty, fuzziness, and vagueness and incorporates qualita- 
tive/quantitative attributes in an integrated framework using belief structures, belief matrices, and a rule/utility-based grading strategy for aggregating the information. The influential feature of this method is that varieties of data can consistently be modelled in an integrated system [33].

There are two general approaches to adopting MCDM in EMOO methods [34]: (a) employing MCDM for Pareto optimal solutions set obtained by EMOO; or (b) integrating MCDM with EMOO as a robust parallel searching means.

Decision-making is widely gone through in several aspects of engineering and some studies have employed MCDM techniques to determine the most appropriate Pareto solutions in project scheduling problems [21], building design [22], water distribution systems [28], etc. However, in most of the appliance scheduling problems, only Pareto solutions are acquired, plotted, and informed. This was one of the reasons that the authors of the present paper were persuaded to employ MCDM techniques in solving MOHASP to determine the best appliance operation schedule of a given smart home. Except for the paper of Du et al. [35], in which a user-oriented weighting approach decided the best schedule, there is no research proposing an exhaustive framework for synthesizing the MCDM techniques with MOO methods to more efficiently schedule the appliances.

Previous studies have investigated the influence of smart home appliances scheduling on their operational unsafety [35], electricity cost of household [35], operational delay of appliances [36], peak-to-average power ratio, and $\mathrm{CO}_{2}$ emission [37]. However, joint optimization of these objectives has been considered in none of the studies. This study tries to optimize all the objectives mentioned above concurrently by assuming that households are equipped with smart appliances. Other new contributions of this paper include a proposed MOHASP modeling framework incorporating an MOALO algorithm, tailored for methodical multicriteria assessment handling, alongside an ER approach for ranking the Pareto solutions. In order to illustrate efficiency and compatibility of the proposed system, an example of home appliance scheduling is solved for identifying the best Pareto solution. The MOALO algorithm is presented in the following sections. First, the Shannon entropy procedure for acquiring the associated relative normalized weights of each objective is explained. Furthermore, the ER approach is elaborated on and the integration framework for the MCDM approaches and for MOO methods is discussed. Then, the formulations and modeling of the MOHASP are given. A benchmark instance from the literature will be solved and the optimal Pareto solutions are identified in order to show the efficiency of the suggested procedure. Given the expectations of the decision makers, the most suitable solution is determined by the ER approach. Efficiency of the results of the suggested methodology is discussed using an identical method and the optimal solution is presented. Finally, concluding remarks and suggestions for future research are provided.

\section{Methodology}

Figure 1 shows the process of the suggested system for energy management beginning by initial adjustment and collection of the necessary data for the real-time day-ahead electricity price, real-time day-ahead $\mathrm{CO}_{2}$ footprint, requirements of the users for the operations of the appliances, at-home and awake statuses of the users, and energy consumption of the appliances. Then, the MOALO algorithm for meeting the global Pareto optimal front is served up. Each objective, consisting of safety, electricity cost, delay, PAR, and $\mathrm{CO}_{2}$ emission, holds a particular corresponding normalized weight. These objectives make employing Shannon's entropy method possible. For the purpose of relating the objectives mentioned above with the overall performance indicator, a hierarchical structure can be formed with the computed corresponding weights to indicate the modality of assessing the overall performance. In the following procedure, the ER technique assists the users in evaluating Pareto solutions (alternatives), specifies overall utility scores, and indicates their level of comfort by each solution while simultaneously considering all the criteria. Finally, the Pareto solutions are sorted according to their utility scores in descending order and the first one is picked as the best solution. The flowchart of the proposed Smart-Home Energy Management System (SHEMS) methodology is presented in Figure 1.

\subsection{Preparing the required information for appliances scheduling operation}

The day-ahead real-time electricity price for tomorrow is passed on from the utility company to the SHEMS. Since users have various demands for the operations of appliances and their at-home statuses and awake statuses are dissimilar, the awake and at-home statuses of the users and their demands for the operations of appliances are put in the SHEMS by the users. The SHEMS provides the schedules for the home appliances by the proposed approach according to the electricity price as well as status and demands of the users. Then, it will automatically control the appliances based on the energy consumption schedules by the home area network $[35,36]$. The smart home appliances are categorized into schedulable, such as washing machines and water heaters, and non-schedulable, such as TV and lights. Energy consumption of schedulable appliances is adjustable and they are schedulable in progress. They are supposed not to be interrupted [35]. The 


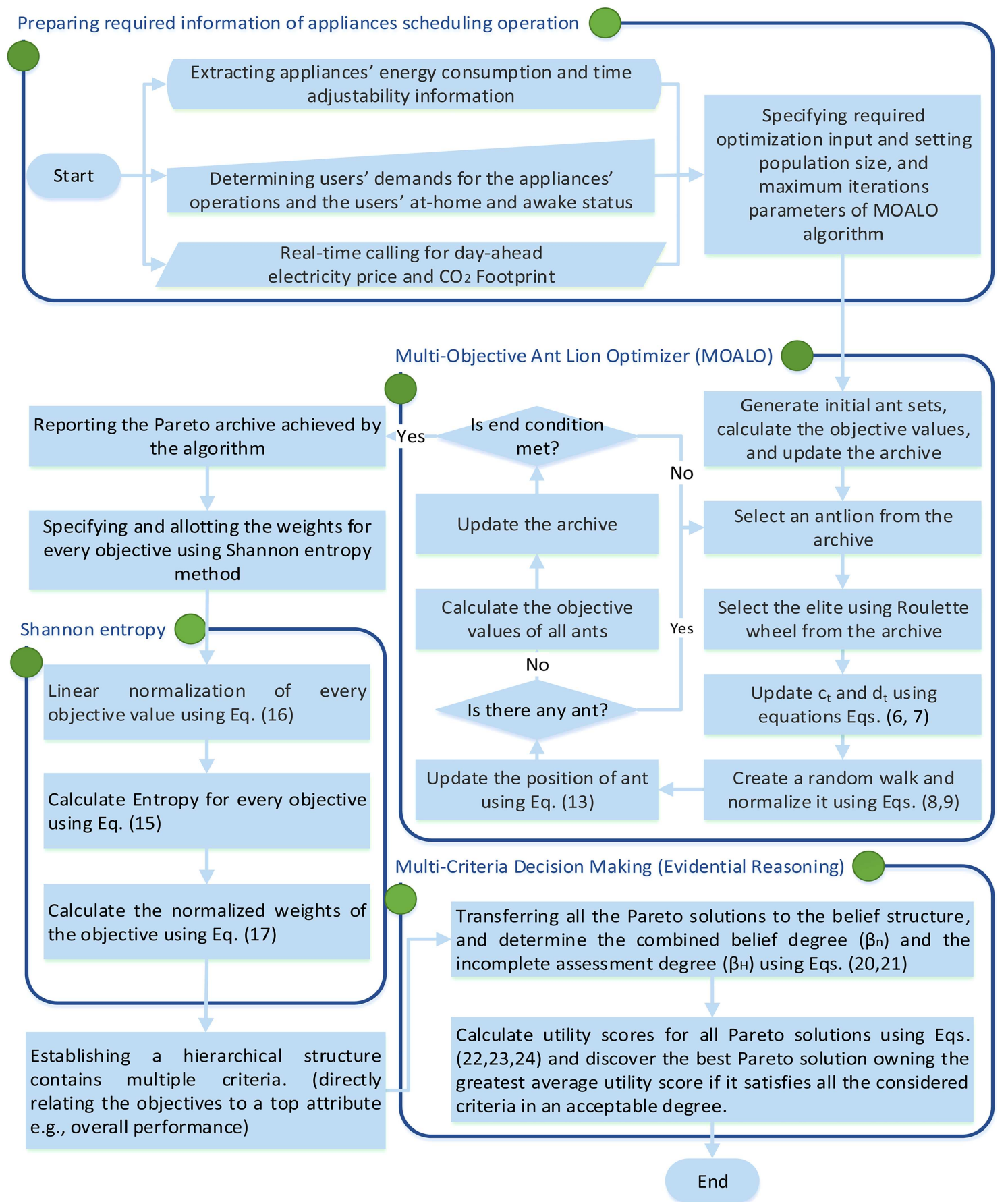

Figure 1. Flowchart of the proposed Smart-Home Energy Management System (SHEMS) methodology.

energy management system will not include the nonschedulable appliances for which the real-time demands of users will be in control of their operation [35]. The information concerning operations of appliances includes the Length of Operation Time (LOT) and the Operation Time Interval (OTI), which are denoted by $\gamma_{a}$ and $\left[\alpha_{a}, \beta_{a}\right]$ for appliance $a$, respectively. Here, $\alpha_{a}$ is the earliest start time and $\beta_{a}$ is the latest finish time of the operation. Regarding the general operation time of appliances, each time slot has 12 minutes and the LOT is assigned to the time slots. The LOT is planned to the least integer greater than or equal to the number when the length of the operation is not an integer multiple of 12 . Therefore, one day is planned in 120 time slots and the OTI is assigned to the related time slot. For example, the LOT is 3, i.e., $\gamma=2$, for an appliance whose LOT is 36 minutes and the OTI is from 1 to 60 , i.e., $\alpha=1 ; \beta=60$ for an appliance whose operation is considered in the range of 12 midnight and 12 noon [36]. 


\subsection{Multi-Objective Optimization (MOO)}

\subsubsection{Basic definitions for $M O O$}

Without loss of generality, the brief fundamentals of MOO (minimization) are defined as follows.

Definition 1 (Pareto dominance). Considering two vectors like $\vec{x}=\left(z_{1}, x_{2}, \ldots, x_{k}\right)$ and $\vec{y}=\left(y_{1}, y_{2}, \ldots, y_{k}\right)$ vector $\vec{x}$ is called to dominate $\vec{y}$ (denoted by $\vec{x} \prec \vec{y}$ ) if and only if [17]:

$$
\begin{aligned}
& \forall i \in\{1,2, \ldots, k\}: f_{i}(\vec{x}) \leq f_{i}(\vec{y}) \wedge \\
& \quad \exists i \in\{1,2, \ldots, k\}: f_{i}(\vec{x})<f_{i}(\vec{y}) .
\end{aligned}
$$

The definition of Pareto optimality is presented as follows $[38,39]$ :

Definition 2 (Pareto optimality). A solution $\vec{x} \in$ $X$ is assumed Pareto optimal if and only if:

$$
\{\nexists \vec{y} \in X \mid \vec{x} \prec \vec{y}\} \text {. }
$$

Definition 3 (Pareto optimal set). The set of all Pareto optimal solutions is defined as follows:

$$
P_{s}:=\{\vec{x}, \vec{y} \in X \mid \nexists \vec{x} \prec \vec{y}\} .
$$

Definition 4 (Pareto optimal front). A set including the values of objective functions for Pareto solutions set is:

$$
P_{f}:=\left\{\overrightarrow{f(x)} \mid \vec{x} \in P_{s}\right\}
$$

2.2.2. Multi-Objective Ant Lion Optimizer (MOALO) MOALO is one of the newest nature-inspired optimization algorithms introduced by Mirjalili et al. [17], which mimics hunting behavior of antlions and their interactions with the favorite corresponding prey-ants. The antlion algorithm has two types of populations, namely the antlions and the ants. The general hunting process of MOALO represents the interaction among antlions and ants in the trap as follows: random walk of ants, building traps, entrapment of ants in traps, catching preys, re-building the traps, and elitism. An antlion larva walks on a circular path and throws out sand by its massive jaw to dig a cone-shape trap by a very sharp edge in the sand so that the insects easily fall to its bottom. Then, the larva hides underneath the bottom of the trap and waits for preys to fall in. If the antlion realizes that a prey has fallen in the trap, it tries to catch the prey and then, pulls it under the soil. After consuming the prey, it throws the leftovers outside the pit, amending the pit for the next catch [12]. In the algorithm, the archive is first used to save non-dominated Pareto optimal solutions obtained so far. Then, solutions are chosen from this archive using a roulette wheel mechanism based on coverage of the solutions (as antlions) to guide ants to promising areas of multi-objective search spaces. Reviewing the literature shows its efficiency in solving challenging real-world problems and it is selected not only due to its solution quality but also for its convergence speed and very small number of parameters. The optimization process of the MOALO algorithm is described below [17]:

Step 1. Initialize the set of ants and antlions with random values.

Step 2. Choose a random antlion from the archive.

Step 3. Choose the elite by Roulette wheel and Eq. (5) from the archive:

$$
P_{i}=\frac{c}{N_{i}}
$$

Step 4. Update $c$ and $d$ by Eqs. (6) and (7):

$$
\begin{aligned}
& c_{t}=\frac{c_{t}}{I}, \\
& d_{t}=\frac{d_{t}}{I},
\end{aligned}
$$

where $c_{t}$ is the minimum of all variables at the $t$ th iteration, $d_{t}$ presents the vector including the maximum of all variables at the $t$ th iteration, and $=1+10^{\omega} \frac{t}{T}$; in this formula, $t$ is the current iteration, $T$ is the maximum number of iterations, and $\omega$ is defined based on the current iteration to adjust the accuracy level of exploration:

$$
\omega= \begin{cases}2 & \text { when } 0.1 T<t \\ 3 & \text { when } 0.5 T<t \\ 4 & \text { when } 0.75 T<t \\ 5 & \text { when } 0.9 T<t \\ 6 & \text { when } 0.95 T<t\end{cases}
$$

Step 5. Create a random walk and its normalization. The ALO algorithm uses the following formulation to simulate the random walk of ants:

$$
\begin{aligned}
X(t)= & {\left[0, \operatorname{cumsum}\left(2 r\left(t_{1}\right)-1\right),\right.} \\
& \operatorname{cumsum}\left(2 r\left(t_{2}\right)-1\right), \ldots, \\
& \left.\operatorname{cumsum}\left(2 r\left(t_{3}\right)-1\right)\right] .
\end{aligned}
$$

In this formulation, cumsum computes the cumulative sum, $n$ shows the maximum iteration number, $t$ is the iteration (random walk step in this study), and:

$$
r(t)= \begin{cases}1 & \text { if } \text { rand }>0.5 \\ 0 & \text { if } \text { rand } \leq 0.5\end{cases}
$$

is a stochastic function in which rand is a random number produced with uniform distribution in the 
range of $[0,1]$. The random walks should be normalized by Eq. (10) to prevent ants from overshooting and to keep random walks in the boundaries of the search space:

$$
\begin{aligned}
& X_{i}^{t}=\frac{\left(X_{i}^{t}-a_{i}\right) \times\left(d_{i}^{t}-c_{i}^{t}\right)}{\left(b_{i}-a_{i}\right)}+c_{i}^{t}, \\
& c_{i}^{t}=\text { Antlion }_{j}^{t}+c^{t}, \\
& d_{i}^{t}=\text { Antlion }_{j}^{t}+d^{t},
\end{aligned}
$$

where $c_{i}^{t}$ presents the minimum of all variables for the $i$ th ant, $d_{i}^{t}$ is the maximum of all variables for the $i$ th ant, and Antlion ${ }_{j}^{t}$ shows the position of the selected $j$ th antlion at the $t$ th iteration.

Step 6. Update position of the ant using Eq. (13):

$$
A n t_{i}^{t}=\frac{R_{A}^{t} \times R_{E}^{t}}{2}
$$

where $A n t_{i}^{t}$ shows the position of the $i$ th ant at the $t$ th iteration, $R_{A}^{t}$ represents random walk around the antlion chosen using the roulette wheel at the $t$ th iteration, and $R_{E}^{t}$ is the random walk around the elite at the $t$ th iteration.

Step 7. If every ant has been traversed, then go to Step 8; otherwise, go to Step 2.

Step 8. Calculate the objective values of all the ants.

Step 9. Update the archive.

Step 10. If the archive is full, eliminate some solutions by roulette wheel and Eq. (14) from the archive to accommodate the new solutions.

$$
P_{i}=\frac{N_{i}}{c} .
$$

Step 11. Check whether the termination condition is met or not. If the condition is met, then go to Step 12; otherwise, go to Step 2.

Step 12. Output the Pareto optimal solutions.

Pareto optimal solutions can be obtained and ranked in various ways using metaheuristic algorithms. MOALO uses an archive to store Pareto optimal solutions and its convergence is inherited from ALO algorithm. Once a solution has been selected from the archive, the ALO algorithm is used in order to improve its quality. Nonetheless, finding the Pareto optimal solutions set with a great variety is a challenging task. To overcome this challenge, the MOPSO based leader selection and archive maintenance strategies are employed. Of particular importance is providing a limit for the archive and to increase the distribution, solutions should be selected from the archive. Distribution of the solutions in the archive is measured by the niching technique in which the proximity of every solution is checked upon a prearranged radius. Then, the number of solutions in the proximity is counted and considered as the distribution measure. To improve the distribution of solutions in the archive, two mechanisms identical with those in MOPSO are considered. First, the solutions with the minimum inhabited vicinity are picked as antlions. Eq. (1) is then used to define the probability of picking a solution from the archive. The flowchart of the MOALO algorithm is shown in Figure 1.

\subsection{Multi-Criteria Decision Making (MCDM)} 2.3.1. Shannon's entropy

Several methods can be utilized to discover the normalized weights of objectives, e.g., Shannon's entropy technique [40], AHP [27], ordered weighted averaging [41], and simple additive weighted approach [42]. In this research, weighting of the attributes is based on crude values of optimal Pareto solutions, because, according to the above-mentioned methods, decisions of the users might be insufficient and result in a partial judgment on weights. To evaluate the relative weights, Shannon's entropy method declares the corresponding importance weights of the attributes based on the differentiation amongst data. Thus, Shannon's entropy can present a more reliable measure for the corresponding weights of the objectives in the loss of preferences of the users [21]. Shannon's entropy acts as a measure for the degree of uncertainty in information formulated in terms of probability theory. It is associated with the information source as a measure of uncertainty. The information can be easily defined as objective values. The uncertainty in information is addressed by Shannon's entropy utilizing the theory of probability. The inherent hypothesis is that lower probability of an event shows its higher chance to provide more information by its occurrence, i.e., an objective with a biased distribution offers more relative importance than a sharply peaked one does $[21,43]$. The Shannon's entropy parameter $\left(E_{j}\right)$ of the $j$ th objective is formulated as follows:

$$
\begin{aligned}
& E_{j}=-\frac{\sum_{i=1}^{n} P_{i j} \ln P_{i j}}{\ln n}, \text { where } i \in\{1,2, \ldots, n\} \text { and } \\
& j \in\{1,2, \ldots, m\}, \\
& P_{i j}=\frac{f_{i j}}{\sum_{i=1}^{n} f_{i j}}, \text { where } i \in\{1,2, \ldots, n\} \text { and } \\
& j \in\{1,2, \ldots, m\}, \\
& \omega_{j}=\frac{\left(1-E_{j}\right)}{\sum_{j=1}^{m}\left(1-E_{j}\right)}, \text { where } \sum_{j=1}^{m} \omega_{j}=1
\end{aligned}
$$

where $f_{i j}$ indicates the $j$ th objective function of the $i$ th solution and $P_{i j}$ is the $j$ th linear normalized objective 
of the $i$ th solution, which is utilized to calculate the value of $E_{j}$ for the $j$ th objective. $n$ and $m$ are the number of solutions and number of objectives, respectively. Finally, $\omega_{j}$ shows the corresponding relative normalized weight of the $j$ th solution, which is determined by Eqs. (15) and (16).

\subsection{Evidential Reasoning (ER)}

Decision making is widely used in different fields of engineering and several approaches have been presented and employed in dealing with MCDM problems, e.g., additive utility function approaches [44], outranking approaches [45], and ER [28]. Using MCDM approaches makes user preference criteria controllable and more efficient. Moreover, the data may be easily transferred to the controller. Hence, the daily repetitive and time-consuming procedure of review and action of the schedule can be adjusted.

The ER is a comprehensive approach to integrated investigation of the MCDM problems under various uncertainty types like ignorance and fuzziness jointly [28]. The ER approach comprises all parts of the MCDM framework, employing the belief matrices and the belief structures. The ER information aggregation methodology contains a rule-or-utility-based information transformation procedure concerning different quantitative and qualitative information types under the required circumstances of utility and value equality [21,33].

In MOO, where objectives are frequently conflicting, the Pareto solutions might be so copious and it could be time-consuming to ultimately select an individual compromising solution. The output of MOO algorithms is a set of non-dominated solutions. Every non-dominated solution meets the scheduling objectives to some extent, which requires the utilization of the MCDM methods to pick the most suitable nondominated solution. The MCDM problems handle the procedure of ordering solutions by considering various criteria. Therefore, taking multiple attributes into consideration, non-dominated solutions can be ordered by using the ER approach, which is able to present more efficient and practical appliance scheduling alternatives. The ER approach includes the following steps $[28,46]$ :

1. Identification and analysis of multiple assessment criteria using a comprehensive study of engineering judgments or expert interviews with regard to the weight assigned to each criterion. This step collects and models various kinds of supporting attributes such as qualitative, quantitative, precise numbers, fuzziness, uncertainties, comparison numbers, and belief structures concerning criteria weights and utility by a belief decision matrix. Precise numbers show single or exact values without any uncertainty, whereas interval numbers denote estimates in ranges and belief structures indicate an evaluation as a distribution (for instance, unsafety of a specific alternative is "Good" to a belief degree of $71 \%$ and, at the same time, it can be evaluated to be "Moderate" to a degree of belief of $29 \%$; such an evaluation can be represented as $\{($ Good, 0.71), (Moderate, 0.29$)\}$ and is referred to as a belief structure). When the assessor is not sufficiently sure on the assessment because of the lack of knowledge or evidence, the sum of probabilities is unequal to one (incomplete assessment). Furthermore, when no data is available to assess the performance of an alternative for a criterion, the total belief degree is assumed to be zero in the belief structure;

2. Transformation of different types of assessment degrees into a general framework of judgment by unifying the belief structures employing ruleand-utility-based information transformation procedures so that they can be consistently compared and aggregated. Belief structures should be translated during this step. For instance, 'Very Bad' and 'Very Good' respectively indicate zero and one, and the remaining grades may/may not be uniformly distributed;

3. Employment of the ER formulation and algorithm to agglomerate the assessing information on multiple criteria types to attain the overall assessment of each alternative;

4. Generation of utility scores or utility intervals in the state of the lack of information. Utility-based ranking is able to assess the overall performance of every alternative with respect to all aspects (criteria) jointly using a systematic-rational prioritizing framework, which presents the best schedule for smart home appliances and a schedule that satisfies all preferences of the users. The ultimately selected solution is a trade-off among preferences of the users.

There are various techniques for calculating the weights of every criterion, such as pairwise matching defined by users, Shannon's entropy, and so on. Since the weights denote the relative importance, normalizing the values is more helpful than using absolute values. The normalized values can be calculated by the following equations:

$$
\omega_{i}=\frac{W_{j}}{\sum_{i=1}^{L} W_{j}}, \text { where } i=\{1,2, \ldots, L\},
$$

subject to:

$$
0 \leq \omega_{i} \leq 1, \text { where } \sum_{i=1}^{L} \omega_{i}=1
$$

The linguistic phrases like 'worst,' 'good,' etc. are 


$$
\begin{aligned}
& \beta_{n}=\frac{\Pi_{i=1}^{L}\left(1-\omega_{i} \sum_{j=1, j \neq n}^{N} \beta_{i, j}\right)-\Pi_{i=1}^{L}\left(1-\omega_{i} \sum_{j=1}^{N} \beta_{i, j}\right)}{\sum_{n=1}^{N} \Pi_{i=1}^{L}\left(1-\omega_{i} \sum_{j=1, j \neq n}^{N} \beta_{i j}\right)-(N-1) \Pi_{i=1}^{L}\left(1-\omega_{i} \sum_{j=1}^{N} \beta_{i, j}\right)-\Pi_{i=1}^{L}\left(1-\omega_{i}\right)}, \\
& \beta_{H}=\frac{\Pi_{i=1}^{L}\left(1-\omega_{i} \sum_{j=1}^{N} \beta_{i, j}\right)-\Pi_{i=1}^{L}\left(1-\omega_{i}\right)}{\sum_{n=1}^{N} \Pi_{i=1}^{L}\left(1-\omega_{i} \sum_{j=1, j \neq n}^{N} \beta_{i j}\right)-(N-1) \Pi_{i=1}^{L}\left(1-\omega_{i} \sum_{j=1}^{N} \beta_{i, j}\right)-\Pi_{i=1}^{L}\left(1-\omega_{i}\right)} .
\end{aligned}
$$

Box I

known as grades and their entire set is denoted by $H=$ $\left\{H_{n}, n=1,2, \ldots, N\right\}$ [21]. The analytical format of the ER procedure is able to determine the combined beliefs degree $\beta_{n}$ of the $n$th grade, where $n \in\{1,2, \ldots, N\}$ and $\beta_{H}$ denotes the assessment of incompleteness for the entire set $H$. Contrarily to the recursive ER, the analytical format necessitates no iteration to assess several attributes, hence presenting more flexibility for optimization and assessment [33]. Eqs. (20) and (21) are presented in Box I to calculate $\beta_{n}$ and $\beta_{H}$, where $\beta_{i, j}$ is degree of belief of the $i$ th primary criterion for the $j$ th grade and $N$ is the number of grades in set $H$. Combined belief degrees and incomplete assessment $\left(\beta_{n}\right.$ and $\left.\beta_{H}\right)$ need to be translated into a single utility score for ranking the alternatives. Therefore, it is essential to produce numerical values corresponding to the belief structures:

$$
\begin{aligned}
& u_{\max }=\sum_{n=1}^{N-1} \beta_{n} u\left(H_{n}\right)+\left(\beta_{n}+\beta_{H}\right) u\left(H_{n}\right), \\
& u_{\min }=\left(\beta_{1}+\beta_{H}\right) u\left(H_{1}\right)+\sum_{n=2}^{N} \beta_{n} u\left(H_{n}\right), \\
& u_{\text {ave }}=\frac{u_{\max }+u_{\text {min }}}{2} .
\end{aligned}
$$

The minimum, maximum, and average values of utility scores are denoted by $u_{\max }, u_{\min }$, and $u_{\text {ave }}$, respectively, and $u\left(H_{n}\right)$ is a function showing utility score of the the $n$th grade. For instance, if $n=6$ and all of the grades are equally ranged in $[0,1]$, then $u\left(H_{n}\right)=\{0,0.2,0.4,0.6,0.8,1\}$. According to Eqs. (7) and (8), if there is no incomplete assessment $(b H=0)$, all the three states of minimum, maximum, and average values of utility scores are equal and can be calculated by the following formulation:

$$
u_{\max }=u_{\min }=u_{\text {ave }}=\sum_{n=1}^{N} \beta_{n} \cdot u\left(H_{n}\right) .
$$

\section{Multi-objective home appliance scheduling problem}

The main model in this paper is derived from the multi- objective demand-side scheduling problem, which was presented by Du et al. [35], and smart-home appliance scheduling problem, which was presented by Sou et al. [37]. Du et al. [35] considered the objectives of operational unsafety of appliances, electricity cost of household, and operational delay of appliances. Peak to Average Ratio (PAR) has a common formula and $\mathrm{CO}_{2}$ emission is taken from Sou et al. Objective functions and formulations of the model are presented in the following:

\subsection{Objective functions}

\subsubsection{Operational unsafety of appliances}

The awake and at-home statuses of the users for controlling the operations of the appliances are considered as operational unsafety of the appliances. This objective function decreases the appliance operation in out-of-home or sleeping condition of users and Unsafety Time Rate (UTR) quantifies this situation. The minimization formulation for operational unsafety $\left(f_{1}(x)\right)$ of appliances in a home with $\mathrm{n}$ time-adjustable appliances has been presented by Du et al. [35] as follows:

$$
\begin{aligned}
& \min _{x} f_{1}(x), \\
& f_{1}(x)=\sum_{a=1}^{n} \rho_{a}^{U T R_{a}\left(X_{a}\right)}, \\
& U T R_{a}\left(X_{a}\right)=\frac{\gamma_{a}-S_{a}\left(X_{a}\right)}{\gamma_{a}}, \\
& S_{a}\left(X_{a}\right)=\sum_{t=1}^{T} S_{a}\left(X_{a}, t\right) \cdot M(t) \cdot N(t), \\
& S_{a}\left(X_{a}, t\right)= \begin{cases}1, & t \in\left[X_{a}, X_{a}+\gamma_{a}-1\right] \\
0, & t \in H\left[X_{a}, X_{a}+\gamma_{a}-1\right]\end{cases} \\
& M(t)= \begin{cases}1, & \text { if users are at home } \\
0, & \text { if users are away }\end{cases} \\
& N(t)= \begin{cases}1, & \text { if users are awake } \\
0, & \text { if users are asleep }\end{cases}
\end{aligned}
$$




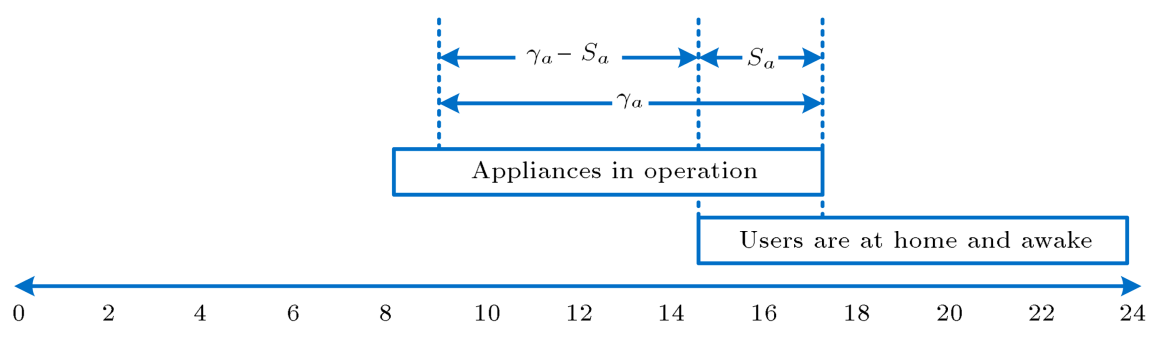

Figure 2. Representation of the Unsafety Time Rate (UTR) concept.

$$
\begin{aligned}
& H=\{1,2, \ldots, T\}, X_{a} \in\left[\alpha_{a}, \beta_{a} \gamma_{a}+1\right] \\
& X=\left\{X_{1}, X_{2}, \ldots, X_{a}, \ldots, X_{n}\right\}
\end{aligned}
$$

subject to:

$$
X_{a} \in\left[\alpha_{a}, \beta_{a}-\gamma_{a}+1\right], \text { where } a=\{1,2, \ldots, n\} .
$$

Within these formulations, UTR of appliance $a$ is denoted by $U T R_{a}$ and $X_{a}$ is the starting time slot of the operation of the appliance. $\rho_{a}$ is the unsafety parameter; with higher values of $\rho_{a}$, the cost of operational unsafety will be higher. The set $X=\left\{X_{1}, X_{2}, \ldots, X_{a}, \ldots, X_{n}\right\}$ indicates the starting time slots of appliances. $S_{a}$ is the number of time slots that users are awake and at home during the time appliance $a$ is in operation and it is determined by the operation status of the appliance $S_{a}\left(X_{a}, t\right)$ with the information of at-home status $M(t)$ and awake status $N(t)$ of the users in a day. The phrase $t \in H\left[X_{a}, X_{a}+\right.$ $\left.\gamma_{a}-1\right]$ demonstrates that $t$ pertains to $H=\{1,2, \ldots, T\}$ excluding the range within this formulation. UTR of appliance $a$ is denoted by $U T R_{a}$ and $X_{a}$ is the starting time slot of its operation. Also, $\rho_{a}$ is the unsafety parameter. The higher the value of $\rho_{a}$, the higher the cost of operational unsafety will be. The set $X=$ $\left\{X_{1}, X_{2}, \ldots, X_{a}, \ldots, X_{n}\right\}$ indicates starting time slots of the appliances. $S_{a}$ represents the number of time slots that users are awake and at home, while the appliance $a$ is in operation and determined using its operation status $S_{a}\left(X_{a}, t\right)$ with the information of the at-home status $M(t)$ and awake status $N(t)$ of the users in a day. The phrase $t \in H \backslash\left[X_{a}, X_{a}+\gamma_{a}-1\right]$ demonstrates that $t$ pertains to $H=\{1,2, \ldots, T\}$ excluding the range $\left[X_{a}, X_{a}+\gamma_{a}-1\right]$ and $T=120$ is the boundary of scheduling, which intimates the number of time slots forward that the schedule of energy consumption is made for time-adjustable appliances. $X_{a} \in\left[\alpha_{a}, \beta_{a} \gamma_{a}+\right.$ $1]$, because the operation should start ahead of the deadline with at least the length of the operation time. After determining the start time slots of appliances, the UTRs of appliances are calculated by Eqs. (28)(33) and the operational unsafety is calculated by UTR. UTR is the ratio of the unsafe operation time slots (the time slots that users are asleep or away, but the appliance is in operation) to the operation length. Consider that various appliances may have identical $U T R \mathrm{~s}$ and $\rho_{a}$ is presented for distinguishing the operational unsafety of appliances. Also, suppose that both the UTR and $\rho_{a}$ jointly define operational unsafety of the appliance by $\rho_{a}^{U T R_{a}}$. The concept of UTR is depicted in Figure 2.

The at-home status $M(t)$ and awake status $N(t)$ of the users are separately determined by users as various users have various at-home statuses and awake statuses. Based on the predefined at-home and awake statuses of the users, the operational unsafety of appliances is calculated by Eqs. (26)-(35). The operational unsafety of the same energy consumption schedule differs under various statuses of users.

\subsubsection{Electricity cost of household}

$P_{a}$ shows the power of appliance $a$. By considering each one hour as five identical time slots with the fixed energy consumption of $\frac{P_{a}}{5}$ during each time slot, the energy consumption schedule of appliance $a$ is calculated by $[35,36]$ :

$$
E_{a}= \begin{cases}e_{a}^{t} \mid e_{a}^{t}=\frac{P a}{5}, & t \in\left[X_{a}, X_{a}+\gamma_{a}-1\right], \\ e_{a}^{t}=0, & t \in H\left[X_{a}, X_{a}+\gamma_{a}-1\right] \\ H=\{1,2, \ldots, T\}, & X_{a} \in\left[\alpha_{a}, \beta_{a} \gamma_{a}+1\right]\end{cases}
$$

where $e_{a}^{t}$ denotes the energy consumption of appliance $a$ during time slot $t$. Pursuant to the energy consumption of appliances and the day-ahead realtime electricity price, the minimization formulation of electricity cost is:

$$
\begin{aligned}
& \min _{x} f_{2}(x) \\
& f_{2}(x)=\sum_{t=1}^{T} p r c_{t} . l_{t}(x), \\
& l_{t}(x)=\sum_{a=1}^{n} e_{a}^{t}, \\
& X=\left\{X_{1}, X_{2}, \ldots, X_{a}, \ldots, X_{n}\right\},
\end{aligned}
$$

subject to:

$$
X_{a} \in\left[\alpha_{a}, \beta_{a}-\gamma_{a}+1\right], \text { where } a=\{1,2, \ldots, n\},
$$


where $\operatorname{prc}_{t}$ is the real-time electricity price at time slot $t$ and $l_{t}$ is total energy consumption of all the timeadjustable appliances during time slot $t$, which can be obtained after determining the start time slots of all appliances $X$. Energy consumption of every appliance is scheduled by Eq. (36).

\subsubsection{Operational delay of appliances}

Figure 3 illustrates that operational delay of an appliance is the delay time from $\alpha_{a}$, the earliest start time of the operation. The longest delay occurs exactly when the appliance reaches the deadline to complete its operation, i.e., the appliance starts at the time slot $\beta_{a}-\gamma_{a}+1$. For a home including $n$ time-adjustable appliances $[35,36]$, the formulation of operational delay minimization is as follows:

$$
\begin{aligned}
& \min _{x} f_{3}(x) \\
& f_{3}(x)=\sum_{a=1}^{n} \sigma_{a}^{D T R_{a}\left(X_{a}\right)} \\
& \operatorname{DTR}_{a}\left(X_{a}\right)=\frac{x_{a}-\alpha_{a}}{\beta_{a}-\gamma_{a}+1-\alpha_{a}} \\
& X=\left\{X_{1}, X_{2}, \ldots, X_{a}, \ldots, X_{n}\right\}
\end{aligned}
$$

subject to:

$$
X_{a} \in\left[\alpha_{a}, \beta_{a}-\gamma_{a}+1\right], \text { where } a=\{1,2, \ldots, n\},
$$

where $D T R_{a}$ indicates the delay time rate of appliance a. $\sigma_{a}>1$ shows the delay parameter of appliance $a$. The higher the value of $\sigma_{a}$, the higher the cost of operational delay will be [36].

\subsubsection{Peak to Average Ratio (PAR)}

Supporting stability of the entire electricity network is an important issue that can be achieved by minimizing the PAR. PAR is the rate of maximum daily power demand to average daily power demand. Minimization formulation of PAR is presented as follows:

$$
\begin{aligned}
& \min _{x} f_{4}(x), \\
& f_{4}(x)=\frac{\max _{x} l_{t}(x)}{\sum_{t=1}^{T} l_{t}(x)}, \\
& l_{t}(x)=\sum_{a=1}^{n} e_{a}^{t}, \\
& X=\left\{X_{1}, X_{2}, \ldots, X_{a}, \ldots, x_{n}\right\},
\end{aligned}
$$

subject to:

$$
X_{a} \in\left[\alpha_{a}, \beta_{a}-\gamma_{a}+1\right] \text {, where } a=\{1,2, \ldots, n\} .
$$

3.1.5. $\mathrm{CO}_{2}$ emission

During day and night, various renewable and nonrenewable sources with different $\mathrm{CO}_{2}$ footprints are used for electricity generation, leading to a dynamic $\mathrm{CO}_{2}$ emission footprint during hours of a day. By shifting operation of the appliances to the hours of the day with low $\mathrm{CO}_{2}$ emissions, the total $\mathrm{CO}_{2}$ emissions of households will be reduced. The minimization formulation for total $\mathrm{CO}_{2}$ emission is presented as follows:

$$
\begin{aligned}
& \min _{x} f_{5}(x), \\
& f_{5}(x)=\sum_{t=1}^{T} l_{t}(x) \cdot C_{t}, \\
& l_{t}(x)=\sum_{a=1}^{n} e_{a}^{t} \\
& X=\left\{X_{1}, X_{2}, \ldots, X_{a}, \ldots, X_{n}\right\},
\end{aligned}
$$

subject to:

$$
X_{a} \in\left[\alpha_{a}, \beta_{a}-\gamma_{a}+1\right], \text { where } a=\{1,2, \ldots, n\},
$$

where $C_{t}$ is the carbon emission in time slot $t$.

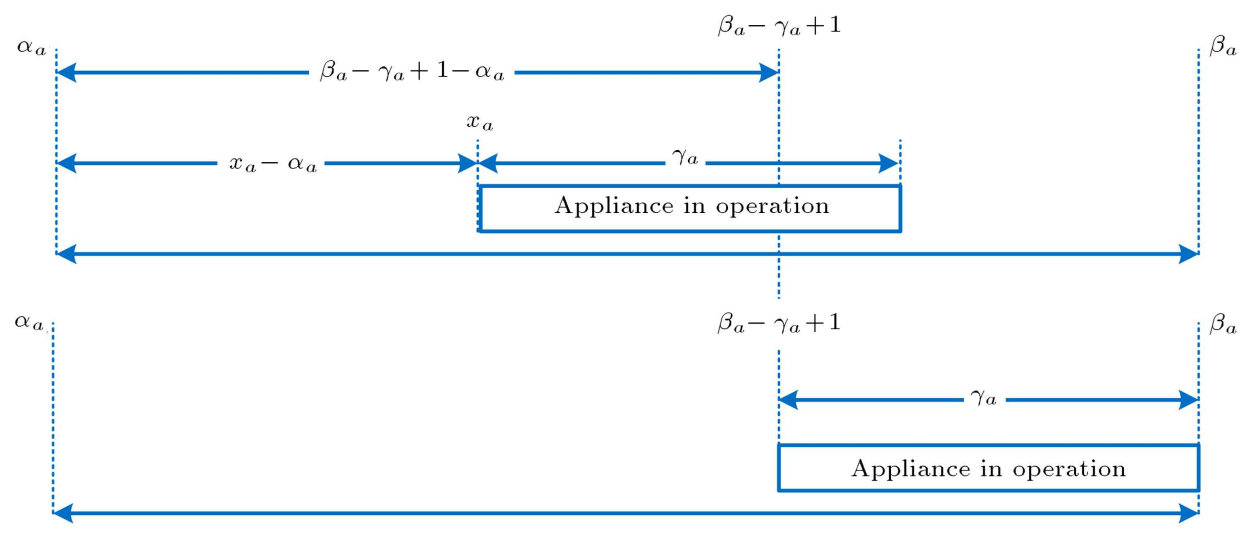

Figure 3. Representation of the delay time rate concept. 


\section{Implementation of the proposed system}

\subsection{Numerical example}

To verify and show effectiveness of the proposed appliance scheduling system in order to unify the ER approach and MOALO for solving SHEMS, a benchmark of smart home, first proposed by Zhao et al. [36], is adopted and the interrelations among different objectives are investigated. Du et al. [35] altered the data to consider the safety of appliances. In this study, eight common appliances were recognized and some of them were used more than once during the day. The corresponding parameters are illustrated in Table 1.

Du et al. [35] considered the at-home and awake statuses of users, as shown in Figures 4 and 5. The electricity price data [47] and $\mathrm{CO}_{2}$ footprint data [48] are shown in Figures 6 and 7 , respectively. Both the unsafety $\left(\rho_{a}\right)$ and delay $\left(\sigma_{a}\right)$ parameters are considered to be 2 . It is worth mentioning that the at-home status and awake status of the users in Figures 4 and 5 are demonstrated to show how statuses of the users are

Table 1. Parameters of appliances.

\begin{tabular}{lccc}
\hline Appliance & $\begin{array}{c}\text { Operation } \\
\text { Time } \\
\text { Interval } \\
\text { (OTI) }\end{array}$ & $\begin{array}{c}\text { Length of } \\
\text { Operation } \\
\text { Time } \\
\text { (LOT) }\end{array}$ & $\begin{array}{c}\text { Power } \\
(\mathbf{k W})\end{array}$ \\
\hline Rice cooker $^{1}$ & $1-40$ & 2 & 0.5 \\
Rice cooker $^{2}$ & $56-65$ & 2 & 0.5 \\
Rice cooker $^{3}$ & $71-90$ & 2 & 0.5 \\
Water heater $_{\text {Dishwasher }}$ & $86-105$ & 3 & 1.5 \\
Washing machine & $101-120$ & 2 & 0.6 \\
Electric kettle $^{1}$ & $1-60$ & 5 & 0.38 \\
Electric kettle $^{2}$ & $81-90$ & 1 & 1.5 \\
Clothes dryer $^{\text {Oven }}$ & $71-90$ & 1 & 1.5 \\
Electric radiator $^{1}$ & $71-90$ & 3 & 0.8 \\
Electric radiator $^{2}$ & $81-65$ & 5 & 1.9 \\
\hline
\end{tabular}

$*^{1}, *^{2}$, and $*^{3}$ indicate that appliance $*$ is

used three times within various OTIs in a day.

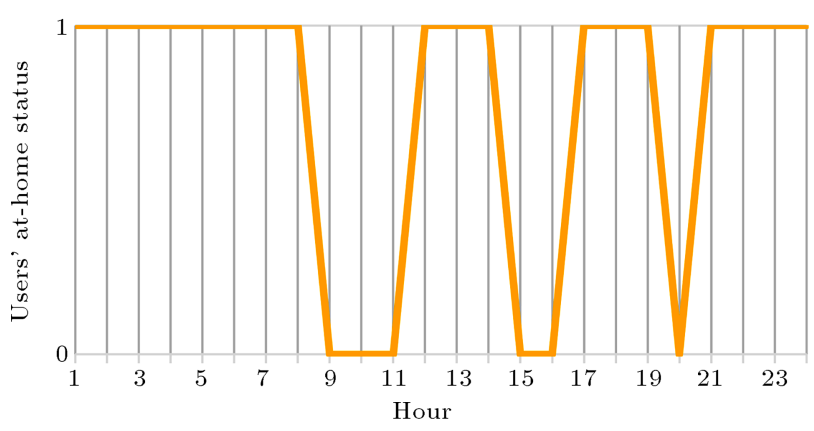

Figure 4. At-home status of the users.

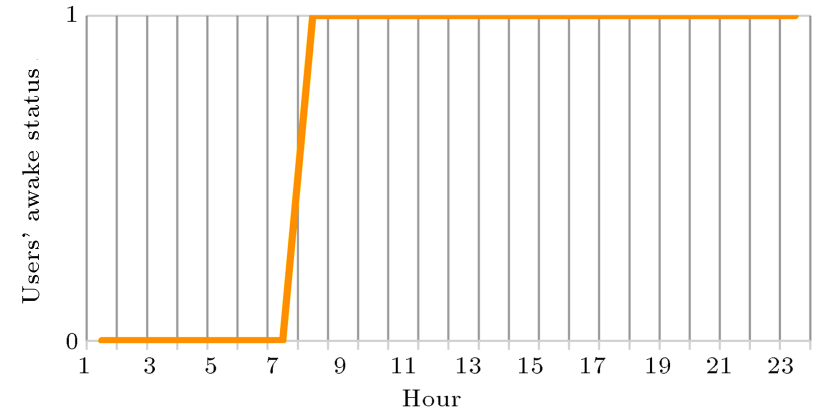

Figure 5. Awake status of the users.

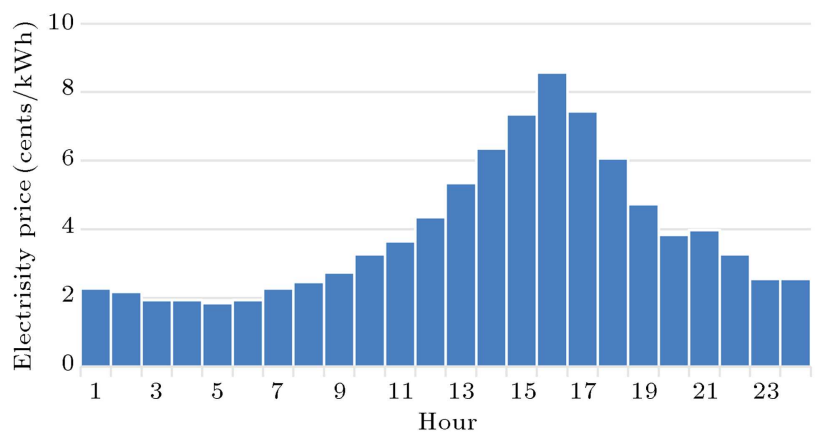

Figure 6. Electricity price data.

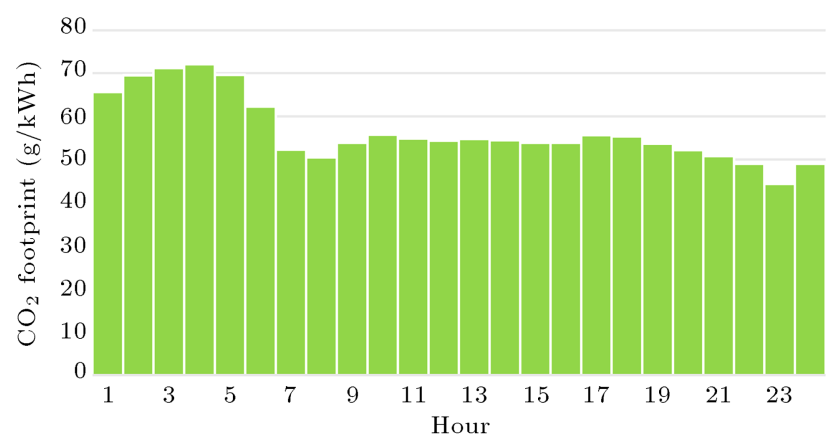

Figure 7. $\mathrm{CO}_{2}$ footprint data.

considered in operational unsafety of the appliances. The users individually define the at-home status and awake status.

Regarding the effect of parameter setting on the performance of metaheuristic algorithms, the Taguchi method [49] is hired. Before calibration of the applied algorithms, some preliminary tests are run to find the proper parameter levels. To achieve more accurate and better sustained results for the proposed algorithm, the population number and iteration parameters are configured.

\subsection{Parameter configuration}

Due to the effect of parameter configuration on the performance of metaheuristics, the Taguchi method of Design-Of-Experiment (DOE) [49] is performed for the configuration of the MOALO parameters. The MOALO contains two key parameters, namely the maximum number of iterations $(T)$ and the num- 
ber of antlions $(n)$. Before calibration of the used algorithms, some preliminary tests are run to find appropriate parameter levels and 5 levels are considered for each of the two parameters. For each run, the maximum number of iterations is set to MaxIt = $\{1000,2000,3000,4000,5000\}$ and the number of ants is set to $n=\{100,150,200,250,300\}$. The performance indicators of multi-objective algorithms differ from single-objective algorithms. In the single-objective case, the optimal solution has the global optimum of a particular objective function, whereas, in the multiobjective case, there may not exist a unique solution that is optimal in terms of all objective functions. Hence, a different method is required for comparing the performance of each test of the algorithm. Therefore, relative performance metrics are used (spread of non-dominance solutions, diversification matrix, mean ideal distance, rate of achievement to two objectives simultaneously, and quality metric) are employed to analyse the results of test runs quantitatively. These metrics are well-known and available in several books and papers. Hence, they are not explained here for brevity. For a thorough explanation of this method and its enhanced version, the interested reader may refer to Jolai et al. [50]. All test results are calculated using the Taguchi method; the mean of S/N (Signal per Noise) ratio is shown in Figure 8. Taguchi is a well-known method of DOE and widely applied in many papers and books. Again, for brevity, it is not repeated in here and for a thorough explanation, the interested reader may refer to [50]. As demonstrated in Figure 8, the second level of iteration numbers and the fourth level

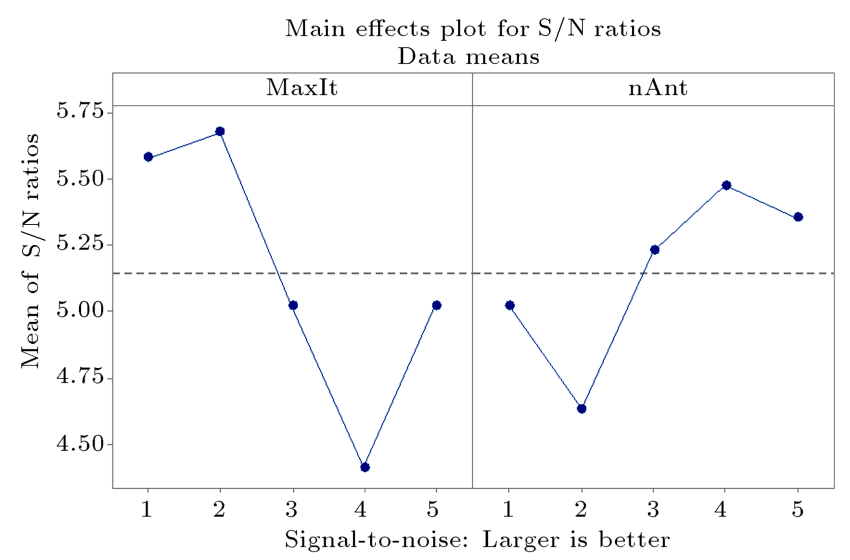

Figure 8. Diagram of the mean effect of the Signal per Noise $(\mathrm{S} / \mathrm{N})$ ratio. of population numbers have better performance. Thus, in this case, the iteration number is set to 2000 and the population number set to 250 .

\subsection{Pareto selection}

Table A.1 (Appendix) presents the acquired Pareto solutions to the benchmark instance of SHEMS. The utility scores of the solutions for each objective are also provided in the table. Figure 9 shows the plot for the parallel coordinates of the Pareto solutions. The data can be used in the prospective research studies. As it can be seen in Figure 10, except for cost-unsafety and $\mathrm{CO}_{2}$ emission-unsafety objective pairs, which have no clear relationship, the relationships between all other objective pairs are oppositional and a Pareto diagram can be plotted for them.

\subsection{Determining the weights}

Shannon's entropy technique determined the weights for all objectives (see Section 2.3.1). The PAR objective possessed the highest value of normalized weight by $41.65 \%$, which for delay, unsafety, cost, and $\mathrm{CO}_{2}$ emission was equal to $36.65 \%, 16.49 \%, 4.91 \%$, and $0.3 \%$, respectively. Table 2 shows that the PAR objective forces a higher impact on the associated uncertainty in the acquired results and possesses a higher weight. It is clear that these weights will change daily by changing the day-ahead $\mathrm{CO}_{2}$ footprint and day-ahead electricity price. This is the advantage of this weighting method over the previously developed methods.

\subsection{Ranking solutions}

The acquired Pareto solutions should now be ranked based on their overall performance, indicating the satisfactory degree of every alternative by taking into account all the criteria simultaneously. In order to assess the overall performance of each solution, a hierarchical structure is required to associate unsafety of the appliances, electricity cost, delay of appliances, PAR, and $\mathrm{CO}_{2}$ emission attributes with their related normalized weights as the indicator of the overall performance, as given in Figure 11. The belief structure of each attribute is determined as demonstrated in Figure 12. The $x$-axis shows the grades ('Worst,' 'Poor,' 'Moderate,' 'Good,' and 'Best') and the corresponding values of the attributes. For example, consider the 40th alternative. The delay of 18.9562 lies between 'Worst' and 'Poor' grades with the values of 22.6271 and 20.0001, respectively. Therefore,

Table 2. Normalized weights of the objectives by Shannon's entropy.

\begin{tabular}{cccccc}
\hline Objectives & Unsafety & Cost & Delay & PAR & CO $_{2}$ emission \\
\hline Normalized weights & 0.1649 & 0.0491 & 0.3665 & 0.4165 & 0.0030 \\
\hline
\end{tabular}




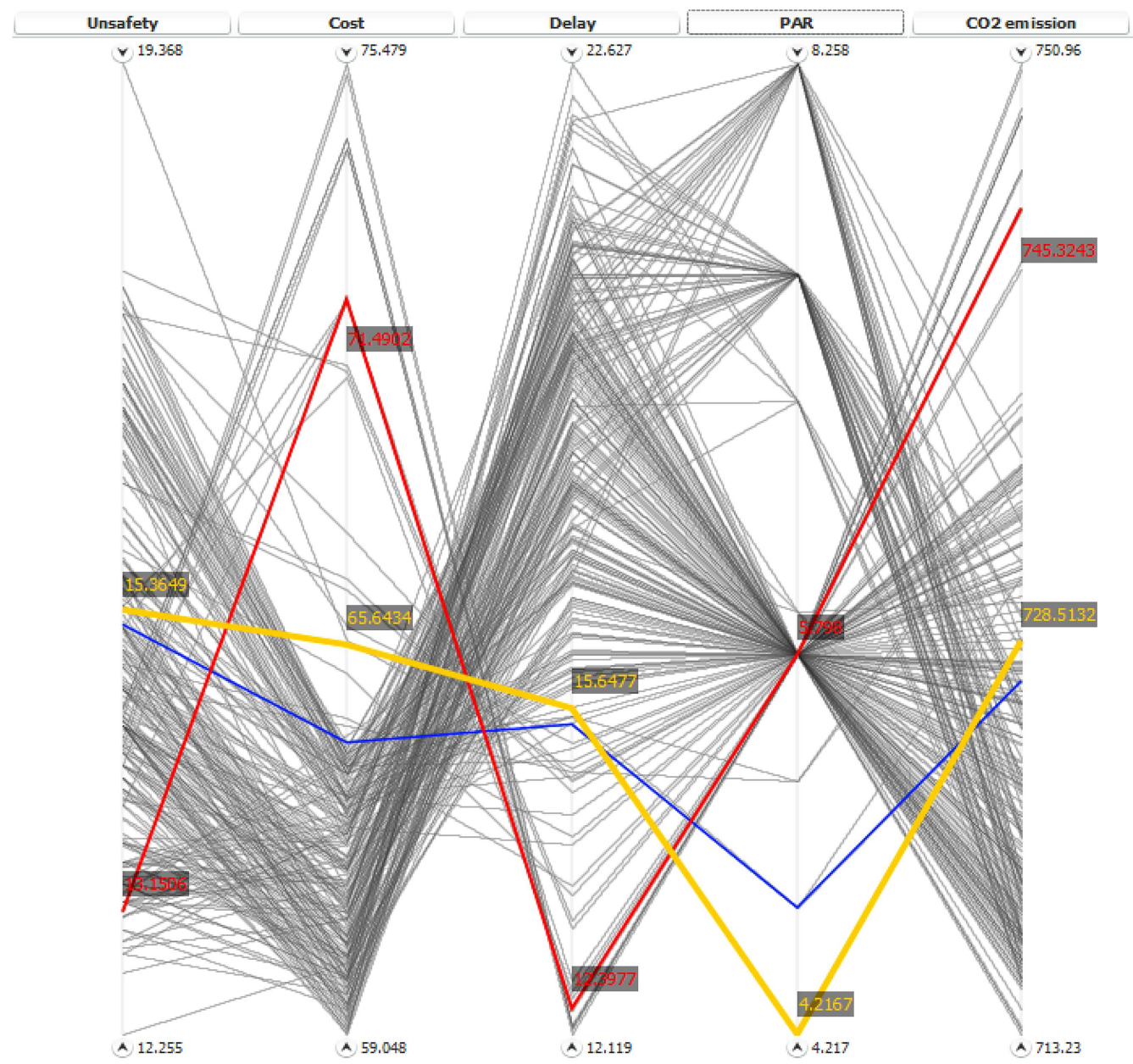

Figure 9. Plot for the parallel coordinates of the Pareto solutions.

Table 3. Belief structures of the attributes of the 40th alternative.

\begin{tabular}{lccccc}
\hline & \multicolumn{5}{c}{ Grades (\%) } \\
\cline { 2 - 6 } & Worst & Poor & Moderate & Good & Best \\
\hline Unsafety & 0 & 0 & 0 & 35.84 & 64.16 \\
Cost & 0 & 0 & 0 & 46.50 & 53.50 \\
Delay & 60.26 & 39.74 & 0 & 0 & 0 \\
PAR & 100 & 0 & 0 & 0 & 0 \\
$\mathbf{C O}_{2}$ emission & 0 & 0 & 0 & 93.22 & 6.78 \\
\hline
\end{tabular}

attribute of the 40 th alternative belongs to the grade of 'Worst' with $60 \%$ belief and to the grade of 'Poor' with $40 \%$ belief. An identical procedure is adopted for all other attributes. The acquired belief structures for all attributes are presented in Table 3. Figure 13 shows the overall performance utility assessment of all solutions. The solutions are sorted based on their acquired overall performance utility scores. The first solution is recommended to the users. In this case, the 6th Pareto solution is selected as the best one because it has the highest utility score (79.52\%) among all the Pareto solutions. That is, this appliance scheduling alternative satisfies the overall performance by concurrently regarding all objectives with the utility score of $79.52 \%$. However, the overall performance might be insufficient for the final decision on the best solution schedule, because every schedule should be reviewed in terms of its weakness and strength points in considering utility score of every objective. As can be observed, the 6th alternative has the best utility score for the PAR objective, which is equal to $100 \%$, and other objectives of this alternative have good levels of utility score. In fact, the ER provides the users with an obvious perception of the performance of each 

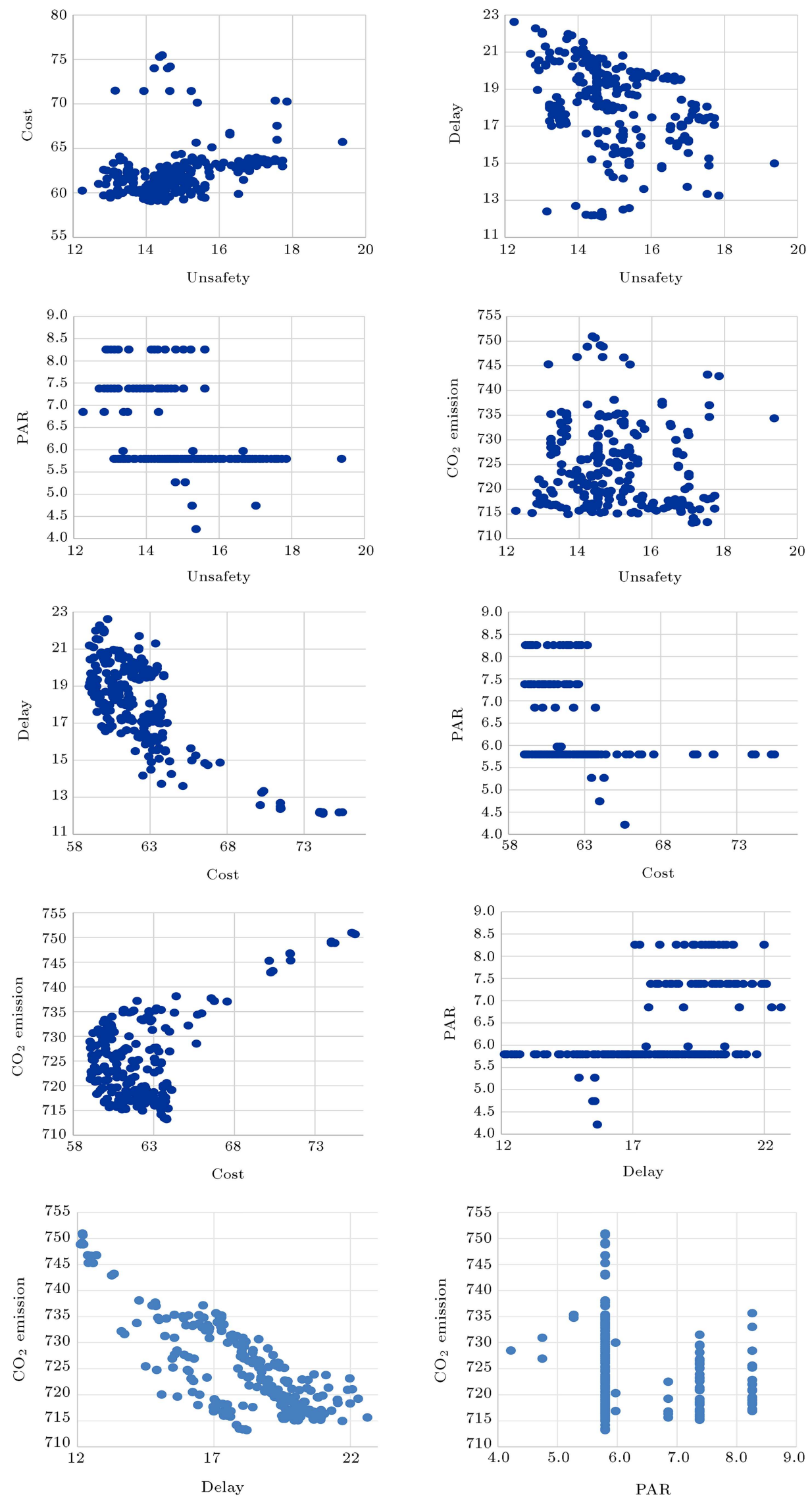

Figure 10. Obtained Pareto optimal solutions shown for each pair of objectives. 


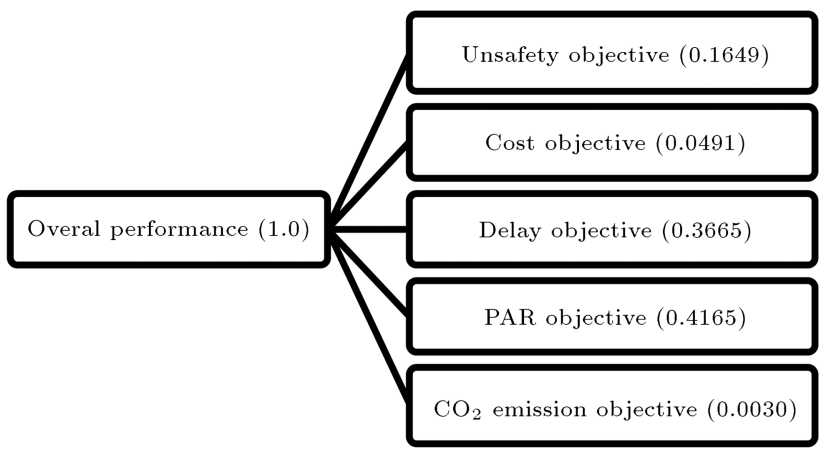

Figure 11. Hierarchical structure of objective weights.

alternative for each single criterion and enables them to investigate schedules quickly.

\subsection{Discussion}

Among the Pareto solutions, the 6th, 40th, 99th, 137th, 181st, and 230th alternatives (Pareto solutions) were chosen to show the procedure of the overall assessment. Figure 14 illustrates the correspondent utility score of every objective along with the overall performance of every alternative. The 181 st alternative possesses $21 \%$ overall utility score, which is quite low to be selected, and does not present satisfactory performance in terms of the unsafety objective. By careful investigation into the best solutions, the users are able to select the most suitable scheduling alternative. ER expedites the investigation into the overall performance of scheduling alternatives by providing more pieces of information about the performance of each scheduling alternative concerning each objective. The presumptions of the users about the performance of each alternative makes them more confident to perform their preferred appliance schedule. Therefore, energy control becomes more efficient in the smart home. As remarked before, the ER approach achieves informative data about the weaknesses of each scheduling alternative at any desirable level. This study divides the overall performance into five grades including 'worst grade,' 'poor grade,' 'moderate grade,' 'good grade,' and 'best grade,' which are spaced equally within the range of $[0,1]$. These grades can be utilized to show the combined degree of belief $\beta_{n}$ (Figure 15). Accordingly, overall performance of the 6 th solution is believed to pertain to the 'best' grade by a degree equal to $79.52 \%$. As well, the 6 th alternative has no belief degree on the 'worst' grade, while the 40th, 137th, and 181st alternatives have belief degrees of the 'worst' grade with the values of $46.05 \%$, $18.10 \%$, and $47.58 \%$, respectively. Consequently, the users decide to select the 6th alternative as the best one. If the first alternative is not suitable, the

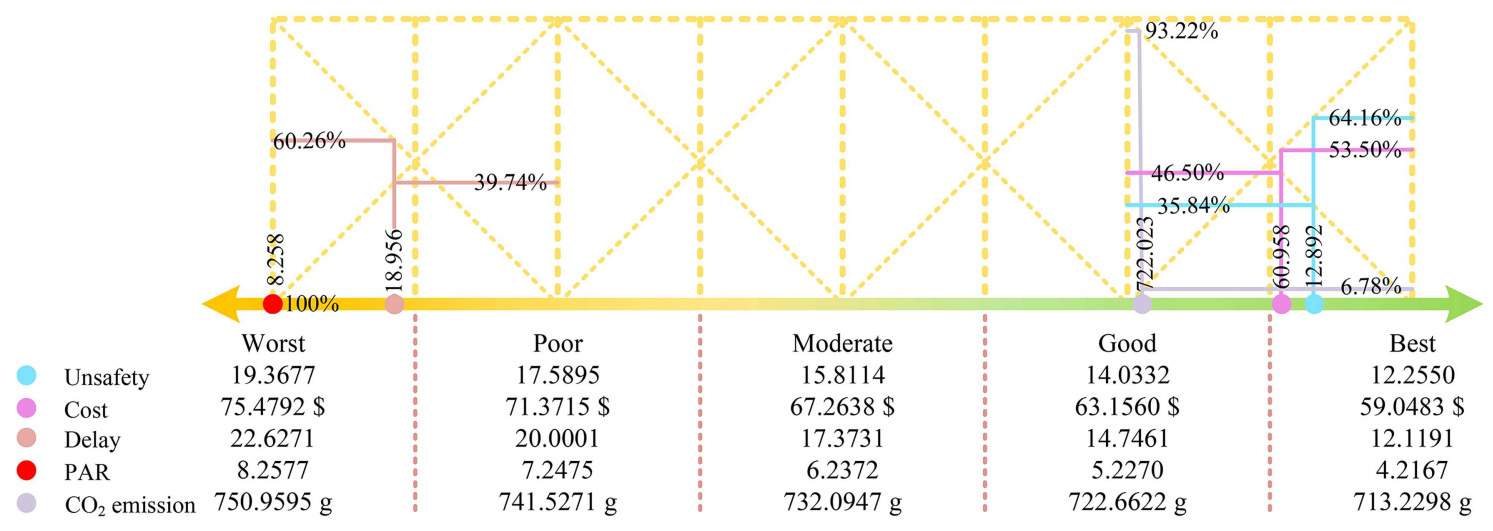

Figure 12. Transfer of each attribute to the belief structure.

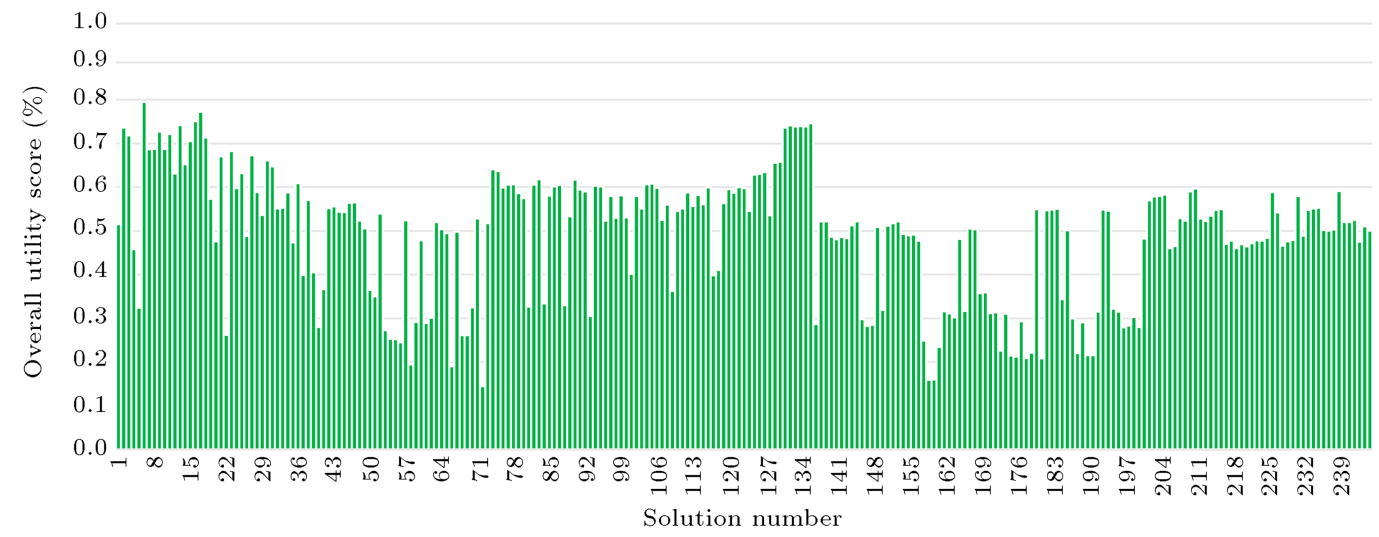

Figure 13. Overall performance utility assessment of all solutions. 


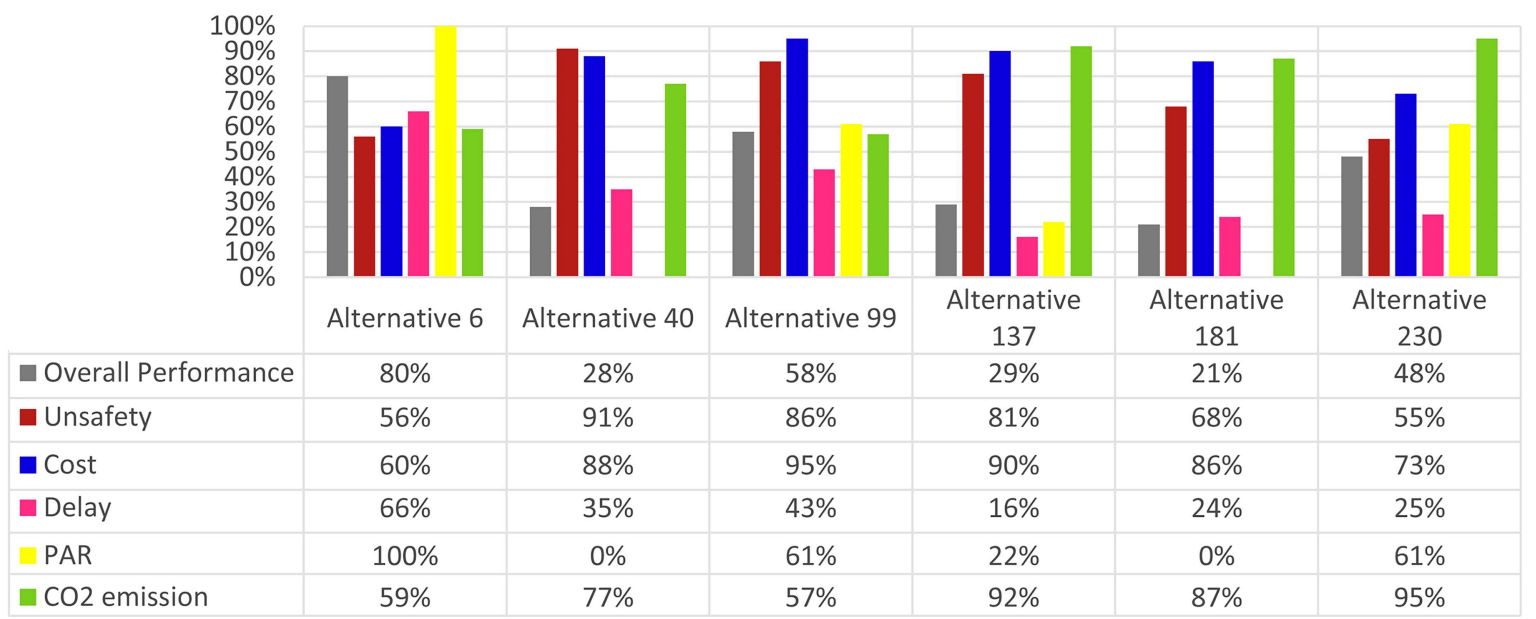

Figure 14. The utility scores of the 6th, 40th, 99th, 137th, 181st, and 230th alternatives concerning each objective.

\begin{tabular}{|c|c|c|c|c|c|c|}
\hline \multirow{2}{*}{$\begin{array}{l}70.00 \% \\
60.00 \%\end{array}$} & & \multicolumn{3}{|c|}{$\square$ Worst $\square$ Poor $\square$ Moderate $\square$ Good $\square$ Best } & & \\
\hline & & & & & & \\
\hline$\stackrel{4}{\Phi} 50.00 \%$ & & & & & & \\
\hline $40.00 \%$ & & & & & & \\
\hline $30.00 \%$ & & & & & & \\
\hline $20.00 \%$ & & & & & & \\
\hline $10.00 \%$ & & & & & & \\
\hline $0.00 \%$ & Alternative 6 & Alternative 40 & Alternative 99 & Alternative 137 & Alternative 181 & Alternative 230 \\
\hline Worst & $0.00 \%$ & $46.05 \%$ & $0.00 \%$ & $18.10 \%$ & $47.58 \%$ & $0.00 \%$ \\
\hline Poor & $0.00 \%$ & $22.49 \%$ & $9.23 \%$ & $67.60 \%$ & $35.98 \%$ & $34.72 \%$ \\
\hline Moderate & $26.10 \%$ & $14.83 \%$ & $56.48 \%$ & $0.00 \%$ & $3.42 \%$ & $38.89 \%$ \\
\hline - Good & $29.70 \%$ & $6.42 \%$ & $26.57 \%$ & $9.86 \%$ & $11.48 \%$ & $26.24 \%$ \\
\hline a Best & $44.20 \%$ & $10.20 \%$ & $7.72 \%$ & $4.44 \%$ & $1.54 \%$ & $0.14 \%$ \\
\hline
\end{tabular}

Figure 15. Combined belief degrees $\left(\beta_{n}\right)$ for the 6 th, 40 th, 99 th, 137 th, 181 st, and 230 th alternatives concerning the overall performance.

users could start investigating other alternatives. In this case, there is no incomplete assessment, so the incompleteness assessment is considered to be zero, $\beta_{H}=0$.

In general, ER is highly effective in determining the performance of each alternative and it empowers the users to make a practical and transparent decision on the best scheduling alternative. Employing the ER procedure in smart home appliance scheduling makes a more efficient electrical energy control system possible and provides the users with higher confidence in their decisions, because they have a transparent knowledge on the performance of each scheduling alternative. In some cases, an inappropriate alternative from the point of view of the users may have the highest value of utility score. In such cases, the next alternative could be investigated.

Concerning the goal of achieving a trade-off, a solution that reaches a balance among unsafety, cost, delay, PAR, and $\mathrm{CO}_{2}$ emission is preferred.
In comparison to other solutions, the 6th solution provides a suitable trade-off among the objectives by concurrently satisfying them to an acceptable degree. The best schedule is obtained in this paper and the relative objectives are shown in Figure 16. If the user recognizes that the schedule cannot satisfy their preference in terms of unsafety or delay of appliances, they are able to change the relative $\rho_{a}$ or $\sigma_{a}$ of the appliance.

\section{Concluding remarks and future works}

In this research, for the first time, unsafety of the appliances, electricity cost, delay of appliances, Peak to Average Ratio (PAR), and $\mathrm{CO}_{2}$ emission were considered as the objectives of a smart home appliance scheduling model and jointly optimized. Moreover, a comprehensive Multi-Criteria Decision Making (MCDM) framework for ranking the acquired Pareto solutions was tailored and evaluated for making 

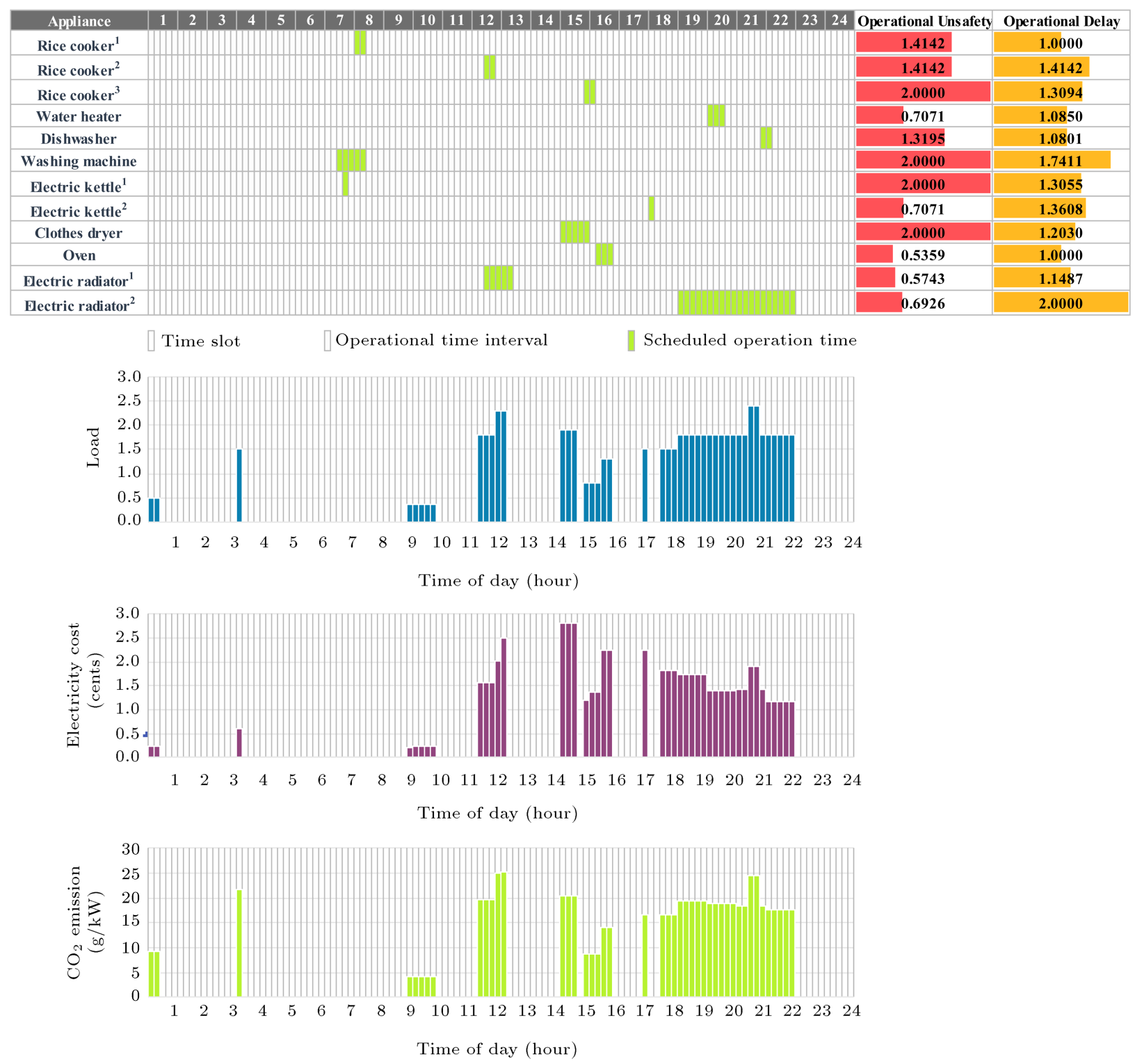

Figure 16. The best solution acquired in this research.

a trade-off between objectives. A Multi-Objective Ant Lion Optimizer (MOALO) was designed to solve Smart-Home Energy Management System (SHEMS) and then, applied to a benchmark appliance scheduling instance from the literature. After that, an Evidential Reasoning (ER) approach was adopted to rank the optimal Pareto solutions employing the weights acquired by Shannon's entropy. The proposed ER approach helps users to identify the efficiency of every alternative in a panoramic view to all the attributes and, consequently, to select one of the Pareto solutions with higher assurance. A comprehensive framework to integrate MCDM techniques into an Evolutionary Multi-Objective Optimization (EMOO) was employed to facilitate the process of making a trade-off among objectives. The proposed approach to employing the
MCDM technique made it possible to select the most efficient appliance schedule. The ER approach gives a transparent and comprehensive sense of efficiency of the alternatives and the users can find the strengths and weaknesses of each alternative. The implementation of the developed SHEMS will need easy access to the data of day-ahead electricity cost and $\mathrm{CO}_{2}$ emission.

In future works on the basis of this study, the authors intend to provide a user-friendly API utilizing social media and BIM to help automatize the process of smart home appliance operation scheduling. Considering the multi-user nature of smart homes, integrating the developed system with game theory concepts may be beneficial. Testing several metaheuristics for solving this problem will also be an interesting topic. 


\section{References}

1. Iwafune, Y. and Yagita, Y. "High-resolution determinant analysis of Japanese residential electricity consumption using home energy management system data", Energy Build., 116, pp. 274-284 (2016).

2. Nejat, P., Jomehzadeh, F., Taheri, M.M., et al. "A global review of energy consumption, $\mathrm{CO} 2$ emissions and policy in the residential sector (with an overview of the top ten CO2 emitting countries)", Renew. Sustain. Energy Rev., 43, pp. 843-862 (2015).

3. Geng, Y., Chen, W., Liu, Z., et al. "A bibliometric review: Energy consumption and greenhouse gas emissions in the residential sector", J. Clean. Prod., 159(800), pp. 301-316 (2017).

4. Naji, H.I., Mahmood, M., and Mohammad, H.E. "Using BIM to propose building alternatives towards lower consumption of electric power in Iraq", Asian J. Civ. Eng., 20(5), pp. 669-679 (2019).

5. Kaveh, A., Shamsapour, N., Sheikholeslami, R., et al. "Forecasting transport energy demand in Iran using meta-heuristic algorithms", Int. J. Optim. Civ. Eng., 2(4), pp. 533-544 (2012).

6. Kaveh, A., Advances in Metaheuristic Algorithms for Optimal Design of Structures, Second Edi, Springer International Publishing, Cham, Switzerland (2017).

7. Kaveh, A. and Vazirinia, Y. "Chaotic vibrating particles system for resource constraint project scheduling problem", Sci. Iran. Trans. A Civ. Eng. (In Press).

8. Golberg, D.E., Genetic Algorithms in Search, Optimization, and Machine Learning, Addison-Wesley Publishing Company, Reading, Massachusetts (1989).

9. Russell, E. and Kennedy, J. "A new optimizer using particle swarm theory", Proc. Sixth Int. Symp. Micro Mach. Hum. Sci., Nagoya, Japan, pp. 39-43 (1995).

10. Kaveh, A. and Mahdavi, V.R. "Resource allocation and time-cost trade-off using colliding bodies optimization", In Colliding Bodies Optimization: Extensions and Applications, Springer International Publishing, Cham, pp. 261-277 (2015).

11. Kaveh, A. and Ilchi Ghazaan, M. "A new metaheuristic algorithm: vibrating particles system", Sci. Iran. Trans., A, Civ. Eng., 24(2), pp. 1-32 (2017).

12. Mirjalili, S. "The ant lion optimizer", Adv. Eng. Softw., 83, pp. 80-98 (2015).

13. Deb, K., Pratap, A., Agarwal, S., et al. "A fast and elitist multiobjective genetic algorithm: NSGA-II", IEEE Trans. Evol. Comput., 6(2), pp. 182-197 (2002).

14. Coello, C.A., Pulido, G.T., and Lechuga, M.S. "Handling multiple objectives with particle swarm optimization", IEEE Trans. Evol. Comput., 8(3), pp. 256-279 (2004).

15. Kaveh, A. and Mahdavi, V.R. "Multi-objective colliding bodies optimization algorithm for design of trusses", J. Comput. Des. Eng., 6(1), pp. 49-59 (2019).
16. Kaveh, A. and Ilchi Ghazaan, M. "A new VPS-based algorithm for multi-objective optimization problems", Eng. With Comput. (In press).

Kaveh, A. and Ilchi Ghazaan, M. "A new VPS-based algorithm for multi-objective optimization problems", Eng. Comput., (0123456789) (2019).

17. Mirjalili, S., Jangir, P., and Saremi, S. "Multiobjective ant lion optimizer: a multi-objective optimization algorithm for solving engineering problems", Appl. Intell., 46(1), pp. 79-95 (2017).

18. Kazemzadeh Azad, S. "Seeding the initial population with feasible solutions in metaheuristic optimization of steel trusses", Eng. Optim., 50(1), pp. 89-105 (2018).

19. Kazemzadeh Azad, S. "Enhanced hybrid metaheuristic algorithms for optimal sizing of steel truss structures with numerous discrete variables", Struct. Multidiscip. Optim., 55(6), pp. 2159-2180 (2017).

20. Mela, K., Tiainen, T., Heinisuo, M., et al. "Comparative study of multiple criteria decision making methods for building design", Adv. Eng. Informatics, 26(4), pp. 716-726 (2012).

21. Monghasemi, S., Nikoo, M.R., Khaksar Fasaee, et al. "A novel multi criteria decision making model for optimizing time-cost-quality trade-off problems in construction projects", Expert Syst. Appl., 42(6), pp. 3089-3104 (2015).

22. Mela, K., Tiainen, T., and Heinisuo, M. "Comparative study of multiple criteria decision making methods for building design", Adv. Eng. Informatics, 26(4), pp. 716-726 (2012).

23. Wallenius, J., Fishburn, P.C., Zionts, S., et al. "Multiple criteria decision making, multiattribute utility theory: Recent accomplishments and what lies ahead", Manage. Sci., 54(7), pp. 1336-1349 (2008).

24. Loken, E. "Use of multicriteria decision analysis methods for energy planning problems", Renew. Sustain. Energy Rev., 11(7), pp. 1584-1595 (2007).

25. Hobbs, B.F. and Meier, P.M. "Multicriteria methods for resource planning: An experimental comparison", IEEE Trans. Power Syst., 9(4), pp. 1811-1817 (1994).

26. Belton, V. and Stewart, T.J., Multiple Criteria Decision Analysis, An Integrated Approach, Springer Science \& Business Media (2002).

27. Saaty, T.L. "Decision making with the analytic hierarchy process", Sci. Iran., 9(3), pp. 215-229 (2002).

28. Bazargan-Lari, M.R. "An evidential reasoning approach to optimal monitoring of drinking water distribution systems for detecting deliberate contamination events", J. Clean. Prod., 78, pp. 1-14 (2014).

29. Lai, Y.-J., Liu, T.-Y., and Hwang, C.-L. "TOPSIS for MODM", Eur. J. Oper. Res., 76, pp. 486-500 (1994).

30. Brans, J.P., Vincke, P., and Mareschal, B. "How to select and how to rank projects: The PROMETHEE method", Eur. J. Oper. Res., 24, pp. 228-238 (1986). 
31. Roy, B. "The outranking approach and the foundations of electre methods", Readings Mult. Criteria Decis. Aid, C.A. e Costa, Ed., Springer Berlin Heidelberg, Berlin, Heidelberg, pp. 155-183 (1990).

32. Ishizaka, A. and Nemery, P., Multi-Criteria Decision Analysis: Methods and Software, John Wiley \& Sons (2013).

33. Yang, J.B., Wang, Y.M., Xu, D.L., et al. "The evidential reasoning approach for MADA under both probabilistic and fuzzy uncertainties", Eur. J. Oper. Res., 171(1), pp. 309-343 (2006).

34. Chaudhuri, S. and Deb, K. "An interactive evolutionary multi-objective optimization and decision making procedure", Appl. Soft Comput. J., 10(2), pp. 496-511 (2010).

35. Du, Y.F., Jiang, L., Li, Y.Z., Counsell, J., et al. "Multi-objective demand side scheduling considering the operational safety of appliances", Appl. Energy, 179, pp. 864-874 (2016).

36. Zhao, Z., Lee, W.C., Shin, Y., and Song, K.-B. "An optimal power scheduling method for demand response in home energy management system", IEEE Trans. Smart Grid, 4(3), pp. 1391-1400 (2013).

37. Sou, K.C., Weimer, J., Sandberg, H., et al. "Scheduling smart home appliances using mixed integer linear programming", Proc. IEEE Conf. Decis. Control, pp. 5144-5149 (2011).

38. Ngatchou, P., Zarei, A., and El-Sharkawi, M. "Pareto multi objective optimization", Proc. 13th Int. Conf. Intell. Syst. Appl. to Power Syst., pp. 84-91 (2005).

39. Pareto, V. "Cours d'economie politique", Libr. Droz, 25 (1964).

40. Shannon, C.E. "A mathematical theory of communication”, Bell Syst. Tech. J., 27(3), pp. 379-423 (1948).

41. Wang, T., Liu, J., Li, J., et al. "An intuitionistic fuzzy OWA-TOPSIS method for collaborative network formation considering matching characteristics", Sci. Iran., 25(3), pp. 1671-1687 (2018).
42. Wang, Y.J. "A fuzzy multi-criteria decision-making model based on simple additive weighting method and relative preference relation", Appl. Soft Comput. J., 30, pp. 412-420 (2015).

43. Deng, H., Yeh, C.H., and Willis, R.J. "Inter-company comparison using modified TOPSIS with objective weights", Comput. Oper. Res., 27(10), pp. 963-973 (2000).

44. Keeney, R.L. and Raiffa, H., Decisions with Multiple Objectives: Preferences and Value Trade-Offs, Cambridge University Press (1993).

45. Guitouni, A., Martel, J., and Vincke, P., A framework to Choose a Discrete Multicriterion Aggregation Procedure, Defence Research Establishment Valcatier (1999).

46. $\mathrm{Xu}, \mathrm{D} . \mathrm{L}$. "An introduction and survey of the evidential reasoning approach for multiple criteria decision analysis", Ann. Oper. Res., 195(1), pp. 163-187 (2012).

47. Ameren Illinois Power Company, Real-time pricing for residential customers, https://www. powersmartpricing.org/prices/.

48. Kristinsdóttir, A.R., Stoll, P., Nilsson, A., and Brandt, $\mathrm{N}$. "Description of climate impact calculation methods of the CO2e-signal for the active house project", KTH Royal Institute of Technology (2013).

49. Taguchi, G., Introduction to Quality Engineering: Designing Quality into Products and Processes, Asian Productivity Organization (1986).

50. Jolai, F., Asefi, H., Rabiee, M., et al. "Bi-objective simulated annealing approaches for no-wait two-stage flexible flow shop scheduling problem", Sci. Iran., 20(3), pp. 861-872 (2013).

\section{Appendix}

Pareto solutions of the MOALO for smart home benchmark

Table A.1. All the acquired Pareto solutions for the smart home appliance scheduling benchmark.

\begin{tabular}{|c|c|c|c|c|c|c|c|c|c|c|c|}
\hline \multirow[b]{2}{*}{ \# } & \multicolumn{5}{|c|}{ Objectives } & \multicolumn{6}{|c|}{ Utility scores in terms of } \\
\hline & Unsafety & Cost & Delay & PAR & $\begin{array}{c}\mathrm{CO}_{2} \\
\text { emission }\end{array}$ & $\begin{array}{c}\text { Overall } \\
\text { performance }\end{array}$ & Unsafety & Cost & Delay & PAR & $\begin{array}{c}\mathrm{CO}_{2} \\
\text { emission }\end{array}$ \\
\hline 1 & 17.1417 & 63.4492 & 17.8280 & 5.7980 & 714.1940 & $52 \%$ & $31 \%$ & $73 \%$ & $46 \%$ & $61 \%$ & $97 \%$ \\
\hline 2 & 14.6435 & 71.4542 & 12.3762 & 5.7980 & 746.8006 & $74 \%$ & $66 \%$ & $24 \%$ & $98 \%$ & $61 \%$ & $11 \%$ \\
\hline 3 & 15.2293 & 71.4569 & 12.4987 & 5.7980 & 746.6918 & $72 \%$ & $58 \%$ & $24 \%$ & $96 \%$ & $61 \%$ & $11 \%$ \\
\hline 4 & 13.6921 & 62.2955 & 21.7105 & 5.7980 & 714.9864 & $46 \%$ & $80 \%$ & $80 \%$ & $9 \%$ & $61 \%$ & $95 \%$ \\
\hline 5 & 12.2550 & 60.2432 & 22.6271 & 6.8521 & 715.6594 & $32 \%$ & $100 \%$ & $93 \%$ & $0 \%$ & $35 \%$ & $94 \%$ \\
\hline 6 & 15.3649 & 65.6434 & 15.6477 & 4.2167 & 728.5132 & $80 \%$ & $56 \%$ & $60 \%$ & $66 \%$ & $100 \%$ & $59 \%$ \\
\hline 7 & 14.9617 & 64.3873 & 14.2542 & 5.7980 & 738.1254 & $69 \%$ & $62 \%$ & $68 \%$ & $80 \%$ & $61 \%$ & $34 \%$ \\
\hline 8 & 15.2293 & 62.5355 & 14.1747 & 5.7980 & 733.7811 & $69 \%$ & $58 \%$ & $79 \%$ & $80 \%$ & $61 \%$ & $46 \%$ \\
\hline 9 & 14.7989 & 64.2737 & 14.9529 & 5.2709 & 734.7895 & $73 \%$ & $64 \%$ & $68 \%$ & $73 \%$ & $74 \%$ & $43 \%$ \\
\hline
\end{tabular}


Table A.1. All the acquired Pareto solutions for the smart home appliance scheduling benchmark (continued).

\begin{tabular}{|c|c|c|c|c|c|c|c|c|c|c|c|}
\hline \multirow[b]{2}{*}{ \# } & \multicolumn{5}{|c|}{ Objectives } & \multicolumn{6}{|c|}{ Utility scores in terms of } \\
\hline & Unsafety & Cost & Delay & PAR & $\begin{array}{c}\mathrm{CO}_{2} \\
\text { emission } \\
\end{array}$ & $\begin{array}{c}\text { Overall } \\
\text { performance }\end{array}$ & Unsafety & Cost & Delay & PAR & $\begin{array}{c}\mathrm{CO}_{2} \\
\text { emission }\end{array}$ \\
\hline 10 & 14.8493 & 63.0491 & 14.5018 & 5.7980 & 725.4890 & $69 \%$ & $64 \%$ & $76 \%$ & $77 \%$ & $61 \%$ & $68 \%$ \\
\hline 11 & 17.0116 & 63.9736 & 15.5519 & 4.7438 & 730.9634 & $72 \%$ & $33 \%$ & $70 \%$ & $67 \%$ & $87 \%$ & $53 \%$ \\
\hline 12 & 15.2226 & 63.5840 & 15.6671 & 5.7980 & 719.6484 & $63 \%$ & $58 \%$ & $72 \%$ & $66 \%$ & $61 \%$ & $83 \%$ \\
\hline 13 & 13.9364 & 71.4542 & 12.6965 & 5.7980 & 746.8006 & $74 \%$ & $76 \%$ & $24 \%$ & $95 \%$ & $61 \%$ & $11 \%$ \\
\hline 14 & 15.3974 & 63.5847 & 15.0823 & 5.7980 & 720.0563 & $65 \%$ & $56 \%$ & $72 \%$ & $72 \%$ & $61 \%$ & $82 \%$ \\
\hline 15 & 15.0665 & 63.4514 & 15.5510 & 5.2709 & 735.3550 & $71 \%$ & $60 \%$ & $73 \%$ & $67 \%$ & $74 \%$ & $41 \%$ \\
\hline 16 & 15.2570 & 63.9868 & 15.4740 & 4.7438 & 726.9526 & $75 \%$ & $58 \%$ & $70 \%$ & $68 \%$ & $87 \%$ & $64 \%$ \\
\hline 17 & 13.1506 & 71.4902 & 12.3977 & 5.7980 & 745.3243 & $77 \%$ & $87 \%$ & $24 \%$ & $97 \%$ & $61 \%$ & $15 \%$ \\
\hline 18 & 15.3970 & 70.1594 & 12.5776 & 5.7980 & 745.2823 & $71 \%$ & $56 \%$ & $32 \%$ & $96 \%$ & $61 \%$ & $15 \%$ \\
\hline 19 & 15.1382 & 61.8578 & 17.1284 & 5.7980 & 718.4045 & $57 \%$ & $59 \%$ & $83 \%$ & $52 \%$ & $61 \%$ & $86 \%$ \\
\hline 20 & 13.1063 & 63.3554 & 21.3051 & 5.7980 & 716.9784 & $48 \%$ & $88 \%$ & $74 \%$ & $13 \%$ & $61 \%$ & $90 \%$ \\
\hline 21 & 16.9856 & 63.7382 & 13.7257 & 5.7980 & 731.6613 & $67 \%$ & $33 \%$ & $71 \%$ & $85 \%$ & $61 \%$ & $51 \%$ \\
\hline 22 & 13.0207 & 59.9607 & 22.0699 & 7.3792 & 721.0676 & $26 \%$ & $89 \%$ & $94 \%$ & $5 \%$ & $22 \%$ & $79 \%$ \\
\hline 23 & 15.7953 & 65.1334 & 13.6117 & 5.7980 & 732.1948 & $68 \%$ & $50 \%$ & $63 \%$ & $86 \%$ & $61 \%$ & $50 \%$ \\
\hline 24 & 14.7750 & 62.6034 & 16.7423 & 5.7980 & 723.3095 & $60 \%$ & $65 \%$ & $78 \%$ & $56 \%$ & $61 \%$ & $73 \%$ \\
\hline 25 & 15.3871 & 63.1638 & 15.5615 & 5.7980 & 727.7293 & $63 \%$ & $56 \%$ & $75 \%$ & $67 \%$ & $61 \%$ & $62 \%$ \\
\hline 26 & 13.2207 & 62.2586 & 20.9829 & 5.7980 & 719.8696 & $49 \%$ & $86 \%$ & $80 \%$ & $16 \%$ & $61 \%$ & $82 \%$ \\
\hline 27 & 14.3759 & 62.9120 & 15.2081 & 5.7980 & 731.2745 & $67 \%$ & $70 \%$ & $76 \%$ & $71 \%$ & $61 \%$ & $52 \%$ \\
\hline 28 & 15.7184 & 62.3380 & 16.4147 & 5.7980 & 718.0575 & $59 \%$ & $51 \%$ & $80 \%$ & $59 \%$ & $61 \%$ & $87 \%$ \\
\hline 29 & 17.7241 & 62.9968 & 17.0731 & 5.7980 & 718.7073 & $54 \%$ & $23 \%$ & $76 \%$ & $53 \%$ & $61 \%$ & $85 \%$ \\
\hline 30 & 15.3974 & 63.0814 & 14.9029 & 5.7980 & 724.7709 & $66 \%$ & $56 \%$ & $75 \%$ & $74 \%$ & $61 \%$ & $69 \%$ \\
\hline 31 & 14.9617 & 62.0403 & 15.4956 & 5.7980 & 725.2368 & $65 \%$ & $62 \%$ & $82 \%$ & $68 \%$ & $61 \%$ & $68 \%$ \\
\hline 32 & 14.8214 & 62.9751 & 18.0663 & 5.7980 & 717.8952 & $55 \%$ & $64 \%$ & $76 \%$ & $43 \%$ & $61 \%$ & $88 \%$ \\
\hline 33 & 16.5320 & 63.5697 & 17.0100 & 5.7980 & 717.1116 & $55 \%$ & $40 \%$ & $72 \%$ & $53 \%$ & $61 \%$ & $90 \%$ \\
\hline 34 & 13.2907 & 63.6540 & 17.6197 & 5.7980 & 716.8579 & $59 \%$ & $85 \%$ & $72 \%$ & $48 \%$ & $61 \%$ & $90 \%$ \\
\hline 35 & 13.3738 & 63.7130 & 17.5972 & 6.8521 & 716.8148 & $47 \%$ & $84 \%$ & $72 \%$ & $48 \%$ & $35 \%$ & $90 \%$ \\
\hline 36 & 13.2768 & 64.1006 & 17.0175 & 5.7980 & 719.1450 & $61 \%$ & $86 \%$ & $69 \%$ & $53 \%$ & $61 \%$ & $84 \%$ \\
\hline 37 & 13.5136 & 61.6662 & 17.8799 & 7.3792 & 725.0781 & $40 \%$ & $82 \%$ & $84 \%$ & $45 \%$ & $22 \%$ & $69 \%$ \\
\hline 38 & 13.5136 & 61.5659 & 18.3079 & 5.7980 & 723.5365 & $57 \%$ & $82 \%$ & $85 \%$ & $41 \%$ & $61 \%$ & $73 \%$ \\
\hline 39 & 14.3358 & 61.0696 & 18.9242 & 6.8521 & 722.5242 & $41 \%$ & $71 \%$ & $88 \%$ & $35 \%$ & $35 \%$ & $75 \%$ \\
\hline 40 & 12.8923 & 60.9583 & 18.9562 & 8.2577 & 722.0227 & $28 \%$ & $91 \%$ & $88 \%$ & $35 \%$ & $0 \%$ & $77 \%$ \\
\hline 41 & 14.5812 & 60.7093 & 18.2616 & 7.3792 & 726.5390 & $37 \%$ & $67 \%$ & $90 \%$ & $42 \%$ & $22 \%$ & $65 \%$ \\
\hline 42 & 14.2213 & 60.4876 & 18.6142 & 5.7980 & 726.1011 & $55 \%$ & $72 \%$ & $91 \%$ & $38 \%$ & $61 \%$ & $66 \%$ \\
\hline 43 & 14.6813 & 61.8451 & 18.0378 & 5.7980 & 723.8403 & $56 \%$ & $66 \%$ & $83 \%$ & $44 \%$ & $61 \%$ & $72 \%$ \\
\hline 44 & 14.2213 & 61.5587 & 18.8636 & 5.7980 & 720.6617 & $54 \%$ & $72 \%$ & $85 \%$ & $36 \%$ & $61 \%$ & $80 \%$ \\
\hline 45 & 14.2213 & 61.4180 & 18.8964 & 5.7980 & 721.1667 & $54 \%$ & $72 \%$ & $86 \%$ & $36 \%$ & $61 \%$ & $79 \%$ \\
\hline 46 & 13.4135 & 61.6166 & 18.5765 & 5.7980 & 721.4916 & $56 \%$ & $84 \%$ & $84 \%$ & $39 \%$ & $61 \%$ & $78 \%$ \\
\hline 47 & 13.9742 & 61.8152 & 18.2966 & 5.7980 & 721.8166 & $57 \%$ & $76 \%$ & $83 \%$ & $41 \%$ & $61 \%$ & $77 \%$ \\
\hline 48 & 15.3923 & 60.5147 & 18.7253 & 5.7980 & 722.8884 & $52 \%$ & $56 \%$ & $91 \%$ & $37 \%$ & $61 \%$ & $74 \%$ \\
\hline 49 & 14.6363 & 59.5679 & 19.8617 & 5.7980 & 721.1451 & $51 \%$ & $67 \%$ & $97 \%$ & $26 \%$ & $61 \%$ & $79 \%$ \\
\hline 50 & 14.7812 & 60.6823 & 18.2502 & 7.3792 & 723.3192 & $37 \%$ & $64 \%$ & $90 \%$ & $42 \%$ & $22 \%$ & $73 \%$ \\
\hline 51 & 14.5136 & 59.4906 & 18.7327 & 7.3792 & 723.3209 & $35 \%$ & $68 \%$ & $97 \%$ & $37 \%$ & $22 \%$ & $73 \%$ \\
\hline 52 & 14.3136 & 59.5317 & 18.9923 & 5.7980 & 722.9183 & $54 \%$ & $71 \%$ & $97 \%$ & $35 \%$ & $61 \%$ & $74 \%$ \\
\hline 53 & 14.1336 & 59.0726 & 21.1977 & 7.3792 & 721.3806 & $27 \%$ & $74 \%$ & $100 \%$ & $14 \%$ & $22 \%$ & $78 \%$ \\
\hline
\end{tabular}


Table A.1. All the acquired Pareto solutions for the smart home appliance scheduling benchmark (continued).

\begin{tabular}{|c|c|c|c|c|c|c|c|c|c|c|c|}
\hline \multirow[b]{2}{*}{$\#$} & \multicolumn{5}{|c|}{ Objectives } & \multicolumn{6}{|c|}{ Utility scores in terms of } \\
\hline & Unsafety & Cost & Delay & PAR & $\begin{array}{c}\mathrm{CO}_{2} \\
\text { emission }\end{array}$ & $\begin{array}{c}\text { Overall } \\
\text { performance }\end{array}$ & Unsafety & Cost & Delay & PAR & $\begin{array}{c}\mathrm{CO}_{2} \\
\text { emission }\end{array}$ \\
\hline 54 & 13.8252 & 60.0048 & 21.9048 & 7.3792 & 720.9801 & $25 \%$ & $78 \%$ & $94 \%$ & $7 \%$ & $22 \%$ & $79 \%$ \\
\hline 55 & 13.7279 & 60.0275 & 21.9785 & 7.3792 & 723.1382 & $25 \%$ & $79 \%$ & $94 \%$ & $6 \%$ & $22 \%$ & $74 \%$ \\
\hline 56 & 14.1336 & 59.5729 & 19.3477 & 8.2577 & 721.0120 & $25 \%$ & $74 \%$ & $97 \%$ & $31 \%$ & $0 \%$ & $79 \%$ \\
\hline 57 & 14.0505 & 59.1530 & 19.6956 & 5.7980 & 722.2769 & $52 \%$ & $75 \%$ & $99 \%$ & $28 \%$ & $61 \%$ & $76 \%$ \\
\hline 58 & 14.3136 & 59.1240 & 20.4415 & 8.2577 & 722.8441 & $19 \%$ & $71 \%$ & $100 \%$ & $21 \%$ & $0 \%$ & $75 \%$ \\
\hline 59 & 13.9279 & 59.9384 & 20.6726 & 7.3792 & 723.9357 & $29 \%$ & $76 \%$ & $95 \%$ & $19 \%$ & $22 \%$ & $72 \%$ \\
\hline 60 & 13.9279 & 59.3325 & 21.1022 & 5.7980 & 723.8097 & $48 \%$ & $76 \%$ & $98 \%$ & $15 \%$ & $61 \%$ & $72 \%$ \\
\hline 61 & 14.3136 & 59.9450 & 20.5745 & 7.3792 & 716.8616 & $29 \%$ & $71 \%$ & $95 \%$ & $20 \%$ & $22 \%$ & $90 \%$ \\
\hline 62 & 14.4110 & 59.4418 & 20.1251 & 7.3792 & 720.8947 & $30 \%$ & $70 \%$ & $98 \%$ & $24 \%$ & $22 \%$ & $80 \%$ \\
\hline 63 & 14.6161 & 62.2988 & 19.3456 & 5.7980 & 719.4611 & $52 \%$ & $67 \%$ & $80 \%$ & $31 \%$ & $61 \%$ & $83 \%$ \\
\hline 64 & 14.4282 & 62.4868 & 19.9747 & 5.7980 & 719.6376 & $50 \%$ & $69 \%$ & $79 \%$ & $25 \%$ & $61 \%$ & $83 \%$ \\
\hline 65 & 15.2777 & 61.3393 & 19.0967 & 5.9736 & 720.3384 & $49 \%$ & $58 \%$ & $86 \%$ & $34 \%$ & $57 \%$ & $81 \%$ \\
\hline 66 & 14.2213 & 59.3630 & 20.5508 & 8.2577 & 720.8116 & $19 \%$ & $72 \%$ & $98 \%$ & $20 \%$ & $0 \%$ & $80 \%$ \\
\hline 67 & 14.1336 & 60.1509 & 20.4066 & 5.7980 & 721.2573 & $50 \%$ & $74 \%$ & $93 \%$ & $21 \%$ & $61 \%$ & $79 \%$ \\
\hline 68 & 14.1336 & 59.5069 & 21.5452 & 7.3792 & 718.4550 & $26 \%$ & $74 \%$ & $97 \%$ & $10 \%$ & $22 \%$ & $86 \%$ \\
\hline 69 & 14.1336 & 59.6788 & 21.5244 & 7.3792 & 719.0413 & $26 \%$ & $74 \%$ & $96 \%$ & $10 \%$ & $22 \%$ & $85 \%$ \\
\hline 70 & 12.8408 & 59.7234 & 22.2827 & 6.8521 & 719.2443 & $33 \%$ & $92 \%$ & $96 \%$ & $3 \%$ & $35 \%$ & $84 \%$ \\
\hline 71 & 13.9645 & 62.4396 & 19.5292 & 5.7980 & 719.9357 & $53 \%$ & $76 \%$ & $79 \%$ & $29 \%$ & $61 \%$ & $82 \%$ \\
\hline 72 & 13.0207 & 59.4680 & 21.9931 & 8.2577 & 718.3342 & $14 \%$ & $89 \%$ & $97 \%$ & $6 \%$ & $0 \%$ & $86 \%$ \\
\hline 73 & 16.6578 & 61.4601 & 17.5023 & 5.9736 & 730.0047 & $52 \%$ & $38 \%$ & $85 \%$ & $49 \%$ & $57 \%$ & $56 \%$ \\
\hline 74 & 16.2847 & 66.7463 & 14.7403 & 5.7980 & 737.1302 & $64 \%$ & $43 \%$ & $53 \%$ & $75 \%$ & $61 \%$ & $37 \%$ \\
\hline 75 & 16.2847 & 66.5647 & 14.8513 & 5.7980 & 737.7221 & $64 \%$ & $43 \%$ & $54 \%$ & $74 \%$ & $61 \%$ & $35 \%$ \\
\hline 76 & 15.1550 & 62.6959 & 16.4547 & 5.7980 & 733.6621 & $60 \%$ & $59 \%$ & $78 \%$ & $59 \%$ & $61 \%$ & $46 \%$ \\
\hline 77 & 15.7036 & 62.8599 & 15.9773 & 5.7980 & 733.3305 & $61 \%$ & $52 \%$ & $77 \%$ & $63 \%$ & $61 \%$ & $47 \%$ \\
\hline 78 & 15.2151 & 62.2222 & 16.2553 & 5.7980 & 733.4252 & $61 \%$ & $58 \%$ & $81 \%$ & $61 \%$ & $61 \%$ & $46 \%$ \\
\hline 79 & 16.5080 & 62.3312 & 16.2180 & 5.7980 & 733.2398 & $59 \%$ & $40 \%$ & $80 \%$ & $61 \%$ & $61 \%$ & $47 \%$ \\
\hline 80 & 15.5877 & 60.5352 & 16.8389 & 5.7980 & 730.8753 & $58 \%$ & $53 \%$ & $91 \%$ & $55 \%$ & $61 \%$ & $53 \%$ \\
\hline 81 & 13.5136 & 62.7928 & 17.2595 & 8.2577 & 733.0318 & $33 \%$ & $82 \%$ & $77 \%$ & $51 \%$ & $0 \%$ & $48 \%$ \\
\hline 82 & 17.5776 & 65.9606 & 15.2584 & 5.7980 & 734.6493 & $61 \%$ & $25 \%$ & $58 \%$ & $70 \%$ & $61 \%$ & $43 \%$ \\
\hline 83 & 17.5776 & 67.5448 & 14.8774 & 5.7980 & 737.0504 & $62 \%$ & $25 \%$ & $48 \%$ & $74 \%$ & $61 \%$ & $37 \%$ \\
\hline 84 & 13.5136 & 63.1664 & 17.0801 & 8.2577 & 735.6544 & $33 \%$ & $82 \%$ & $75 \%$ & $53 \%$ & $0 \%$ & $41 \%$ \\
\hline 85 & 13.2207 & 61.1238 & 18.0882 & 5.7980 & 726.9711 & $58 \%$ & $86 \%$ & $87 \%$ & $43 \%$ & $61 \%$ & $64 \%$ \\
\hline 86 & 13.2207 & 61.7535 & 17.2806 & 5.7980 & 735.2347 & $60 \%$ & $86 \%$ & $84 \%$ & $51 \%$ & $61 \%$ & $42 \%$ \\
\hline 87 & 13.6764 & 60.4028 & 17.1293 & 5.7980 & 733.9633 & $61 \%$ & $80 \%$ & $92 \%$ & $52 \%$ & $61 \%$ & $45 \%$ \\
\hline 88 & 14.0505 & 60.3698 & 19.3792 & 7.3792 & 726.0636 & $33 \%$ & $75 \%$ & $92 \%$ & $31 \%$ & $22 \%$ & $66 \%$ \\
\hline 89 & 14.0505 & 60.5958 & 19.3735 & 5.7980 & 726.1201 & $53 \%$ & $75 \%$ & $91 \%$ & $31 \%$ & $61 \%$ & $66 \%$ \\
\hline 90 & 14.2254 & 61.9653 & 16.6060 & 5.7980 & 737.1763 & $62 \%$ & $72 \%$ & $82 \%$ & $57 \%$ & $61 \%$ & $37 \%$ \\
\hline 91 & 13.4883 & 62.5930 & 17.3593 & 5.7980 & 733.5048 & $59 \%$ & $83 \%$ & $78 \%$ & $50 \%$ & $61 \%$ & $46 \%$ \\
\hline 92 & 19.3677 & 65.7170 & 14.9940 & 5.7980 & 734.3737 & $59 \%$ & $0 \%$ & $59 \%$ & $73 \%$ & $61 \%$ & $44 \%$ \\
\hline 93 & 13.2207 & 61.8930 & 18.0217 & 8.2577 & 728.4742 & $31 \%$ & $86 \%$ & $83 \%$ & $44 \%$ & $0 \%$ & $60 \%$ \\
\hline 94 & 13.6484 & 61.1524 & 17.1547 & 5.7980 & 735.3783 & $60 \%$ & $80 \%$ & $87 \%$ & $52 \%$ & $61 \%$ & $41 \%$ \\
\hline 95 & 14.5441 & 60.3545 & 16.8152 & 5.7980 & 732.2314 & $60 \%$ & $68 \%$ & $92 \%$ & $55 \%$ & $61 \%$ & $50 \%$ \\
\hline 96 & 14.5136 & 59.1926 & 19.4110 & 5.7980 & 725.2773 & $52 \%$ & $68 \%$ & $99 \%$ & $31 \%$ & $61 \%$ & $68 \%$ \\
\hline 97 & 13.3555 & 60.0092 & 18.1028 & 5.7980 & 727.4578 & $58 \%$ & $85 \%$ & $94 \%$ & $43 \%$ & $61 \%$ & $62 \%$ \\
\hline
\end{tabular}


Table A.1. All the acquired Pareto solutions for the smart home appliance scheduling benchmark (continued).

\begin{tabular}{|c|c|c|c|c|c|c|c|c|c|c|c|}
\hline \multirow[b]{2}{*}{$\#$} & \multicolumn{5}{|c|}{ Objectives } & \multicolumn{6}{|c|}{ Utility scores in terms of } \\
\hline & Unsafety & Cost & Delay & PAR & $\begin{array}{c}\mathrm{CO}_{2} \\
\text { emission }\end{array}$ & $\begin{array}{c}\text { Overall } \\
\text { performance }\end{array}$ & Unsafety & Cost & Delay & PAR & $\begin{array}{c}\mathrm{CO}_{2} \\
\text { emission }\end{array}$ \\
\hline 98 & 15.0524 & 60.6945 & 18.7427 & 5.7980 & 724.4386 & $53 \%$ & $61 \%$ & $90 \%$ & $37 \%$ & $61 \%$ & $70 \%$ \\
\hline 99 & 13.2207 & 59.8390 & 18.1093 & 5.7980 & 729.2846 & $58 \%$ & $86 \%$ & $95 \%$ & $43 \%$ & $61 \%$ & $57 \%$ \\
\hline 100 & 14.5136 & 59.0930 & 19.1686 & 5.7980 & 727.6424 & $53 \%$ & $68 \%$ & $100 \%$ & $33 \%$ & $61 \%$ & $62 \%$ \\
\hline 101 & 13.2207 & 60.3757 & 17.9799 & 7.3792 & 729.4188 & $40 \%$ & $86 \%$ & $92 \%$ & $44 \%$ & $22 \%$ & $57 \%$ \\
\hline 102 & 13.2207 & 59.7321 & 18.1703 & 5.7980 & 730.2261 & $58 \%$ & $86 \%$ & $96 \%$ & $42 \%$ & $61 \%$ & $55 \%$ \\
\hline 103 & 14.5136 & 59.7548 & 18.4267 & 5.7980 & 728.5667 & $55 \%$ & $68 \%$ & $96 \%$ & $40 \%$ & $61 \%$ & $59 \%$ \\
\hline 104 & 14.5441 & 60.3401 & 16.6917 & 5.7980 & 732.3622 & $61 \%$ & $68 \%$ & $92 \%$ & $56 \%$ & $61 \%$ & $49 \%$ \\
\hline 105 & 14.5441 & 61.4770 & 16.6261 & 5.7980 & 734.8168 & $61 \%$ & $68 \%$ & $85 \%$ & $57 \%$ & $61 \%$ & $43 \%$ \\
\hline 106 & 13.6484 & 60.2172 & 17.2796 & 5.7980 & 732.2641 & $60 \%$ & $80 \%$ & $93 \%$ & $51 \%$ & $61 \%$ & $50 \%$ \\
\hline 107 & 14.5136 & 59.1566 & 19.3477 & 5.7980 & 726.1765 & $53 \%$ & $68 \%$ & $99 \%$ & $31 \%$ & $61 \%$ & $66 \%$ \\
\hline 108 & 14.8065 & 60.0030 & 17.8386 & 5.7980 & 731.3030 & $56 \%$ & $64 \%$ & $94 \%$ & $46 \%$ & $61 \%$ & $52 \%$ \\
\hline 109 & 14.5136 & 59.4036 & 18.4209 & 7.3792 & 728.1273 & $36 \%$ & $68 \%$ & $98 \%$ & $40 \%$ & $22 \%$ & $61 \%$ \\
\hline 110 & 14.5136 & 59.2375 & 18.6406 & 5.7980 & 730.6881 & $55 \%$ & $68 \%$ & $99 \%$ & $38 \%$ & $61 \%$ & $54 \%$ \\
\hline 111 & 15.5183 & 59.5558 & 17.6075 & 5.7980 & 731.4037 & $55 \%$ & $54 \%$ & $97 \%$ & $48 \%$ & $61 \%$ & $52 \%$ \\
\hline 112 & 13.6484 & 60.0652 & 17.6483 & 5.7980 & 730.7418 & $59 \%$ & $80 \%$ & $94 \%$ & $47 \%$ & $61 \%$ & $54 \%$ \\
\hline 113 & 14.9413 & 60.0796 & 17.8576 & 5.7980 & 729.7118 & $56 \%$ & $62 \%$ & $94 \%$ & $45 \%$ & $61 \%$ & $56 \%$ \\
\hline 114 & 13.3555 & 59.9869 & 18.0087 & 5.7980 & 728.2227 & $58 \%$ & $85 \%$ & $94 \%$ & $44 \%$ & $61 \%$ & $60 \%$ \\
\hline 115 & 14.5136 & 59.7321 & 18.0644 & 5.7980 & 730.9709 & $56 \%$ & $68 \%$ & $96 \%$ & $43 \%$ & $61 \%$ & $53 \%$ \\
\hline 116 & 15.2293 & 61.1173 & 16.4521 & 5.7980 & 735.3054 & $60 \%$ & $58 \%$ & $87 \%$ & $59 \%$ & $61 \%$ & $41 \%$ \\
\hline 117 & 13.5136 & 60.3321 & 17.9319 & 7.3792 & 729.5955 & $40 \%$ & $82 \%$ & $92 \%$ & $45 \%$ & $22 \%$ & $57 \%$ \\
\hline 118 & 13.5136 & 60.4125 & 17.6816 & 7.3792 & 731.5180 & $41 \%$ & $82 \%$ & $92 \%$ & $47 \%$ & $22 \%$ & $52 \%$ \\
\hline 119 & 16.5222 & 59.8713 & 16.8378 & 5.7980 & 732.8086 & $56 \%$ & $40 \%$ & $95 \%$ & $55 \%$ & $61 \%$ & $48 \%$ \\
\hline 120 & 15.2293 & 60.1073 & 16.5709 & 5.7980 & 732.9533 & $60 \%$ & $58 \%$ & $94 \%$ & $58 \%$ & $61 \%$ & $48 \%$ \\
\hline 121 & 15.2293 & 59.9566 & 16.7661 & 5.7980 & 733.3702 & $59 \%$ & $58 \%$ & $94 \%$ & $56 \%$ & $61 \%$ & $47 \%$ \\
\hline 122 & 13.6484 & 61.6413 & 17.2025 & 5.7980 & 734.9969 & $60 \%$ & $80 \%$ & $84 \%$ & $52 \%$ & $61 \%$ & $42 \%$ \\
\hline 123 & 13.6484 & 61.1046 & 17.2761 & 5.7980 & 734.8627 & $60 \%$ & $80 \%$ & $87 \%$ & $51 \%$ & $61 \%$ & $43 \%$ \\
\hline 124 & 14.5136 & 59.5796 & 18.6534 & 5.7980 & 726.5798 & $55 \%$ & $68 \%$ & $97 \%$ & $38 \%$ & $61 \%$ & $65 \%$ \\
\hline 125 & 14.9996 & 62.7050 & 15.8599 & 5.7980 & 735.0816 & $63 \%$ & $61 \%$ & $78 \%$ & $64 \%$ & $61 \%$ & $42 \%$ \\
\hline 126 & 14.5692 & 63.0770 & 16.0686 & 5.7980 & 735.2087 & $63 \%$ & $67 \%$ & $75 \%$ & $62 \%$ & $61 \%$ & $42 \%$ \\
\hline 127 & 14.7320 & 62.7145 & 15.9001 & 5.7980 & 734.6931 & $63 \%$ & $65 \%$ & $78 \%$ & $64 \%$ & $61 \%$ & $43 \%$ \\
\hline 128 & 14.5136 & 59.0483 & 18.9812 & 5.7980 & 728.9206 & $54 \%$ & $68 \%$ & $100 \%$ & $35 \%$ & $61 \%$ & $58 \%$ \\
\hline 129 & 17.8452 & 70.2607 & 13.2550 & 5.7980 & 742.9204 & $66 \%$ & $21 \%$ & $32 \%$ & $89 \%$ & $61 \%$ & $21 \%$ \\
\hline 130 & 17.5270 & 70.3729 & 13.3445 & 5.7980 & 743.2157 & $66 \%$ & $26 \%$ & $31 \%$ & $88 \%$ & $61 \%$ & $21 \%$ \\
\hline 131 & 14.6562 & 74.2133 & 12.1999 & 5.7980 & 748.8843 & $74 \%$ & $66 \%$ & $8 \%$ & $99 \%$ & $61 \%$ & $6 \%$ \\
\hline 132 & 14.3653 & 75.2809 & 12.1832 & 5.7980 & 750.9595 & $74 \%$ & $70 \%$ & $1 \%$ & $99 \%$ & $61 \%$ & $0 \%$ \\
\hline 133 & 14.4361 & 75.4792 & 12.1973 & 5.7980 & 750.6642 & $74 \%$ & $69 \%$ & $0 \%$ & $99 \%$ & $61 \%$ & $1 \%$ \\
\hline 134 & 14.5854 & 74.0150 & 12.1653 & 5.7980 & 749.1795 & $74 \%$ & $67 \%$ & $9 \%$ & $100 \%$ & $61 \%$ & $5 \%$ \\
\hline 135 & 14.6562 & 74.2133 & 12.1191 & 5.7980 & 748.8843 & $74 \%$ & $66 \%$ & $8 \%$ & $100 \%$ & $61 \%$ & $6 \%$ \\
\hline 136 & 14.2205 & 74.0222 & 12.2218 & 5.7980 & 748.8843 & $75 \%$ & $72 \%$ & $9 \%$ & $99 \%$ & $61 \%$ & $6 \%$ \\
\hline 137 & 13.6355 & 60.6206 & 20.9640 & 7.3792 & 716.0881 & $29 \%$ & $81 \%$ & $90 \%$ & $16 \%$ & $22 \%$ & $92 \%$ \\
\hline 138 & 15.4883 & 60.1020 & 18.7093 & 5.7980 & 726.3938 & $52 \%$ & $55 \%$ & $94 \%$ & $37 \%$ & $61 \%$ & $65 \%$ \\
\hline 139 & 15.0741 & 60.5304 & 18.9947 & 5.7980 & 725.3321 & $52 \%$ & $60 \%$ & $91 \%$ & $35 \%$ & $61 \%$ & $68 \%$ \\
\hline 140 & 14.4889 & 59.9507 & 20.5186 & 5.7980 & 716.6109 & $49 \%$ & $69 \%$ & $95 \%$ & $20 \%$ & $61 \%$ & $91 \%$ \\
\hline 141 & 15.1923 & 60.1108 & 20.2116 & 5.7980 & 717.4020 & $48 \%$ & $59 \%$ & $94 \%$ & $23 \%$ & $61 \%$ & $89 \%$ \\
\hline
\end{tabular}


Table A.1. All the acquired Pareto solutions for the smart home appliance scheduling benchmark (continued).

\begin{tabular}{|c|c|c|c|c|c|c|c|c|c|c|c|}
\hline \multirow[b]{2}{*}{$\#$} & \multicolumn{5}{|c|}{ Objectives } & \multicolumn{6}{|c|}{ Utility scores in terms of } \\
\hline & Unsafety & Cost & Delay & PAR & $\begin{array}{c}\mathrm{CO}_{2} \\
\text { emission }\end{array}$ & $\begin{array}{c}\text { Overall } \\
\text { performance }\end{array}$ & Unsafety & Cost & Delay & PAR & $\begin{array}{c}\mathrm{CO}_{2} \\
\text { emission }\end{array}$ \\
\hline 142 & 14.7817 & 60.0392 & 20.3422 & 5.7980 & 716.7063 & $49 \%$ & $64 \%$ & $94 \%$ & $22 \%$ & $61 \%$ & $91 \%$ \\
\hline 143 & 15.7413 & 61.8527 & 19.7089 & 5.7980 & 718.7407 & $48 \%$ & $51 \%$ & $83 \%$ & $28 \%$ & $61 \%$ & $85 \%$ \\
\hline 144 & 15.3270 & 61.0949 & 19.1113 & 5.7980 & 721.7035 & $51 \%$ & $57 \%$ & $88 \%$ & $33 \%$ & $61 \%$ & $78 \%$ \\
\hline 145 & 15.1062 & 60.7093 & 18.9790 & 5.7980 & 721.7106 & $52 \%$ & $60 \%$ & $90 \%$ & $35 \%$ & $61 \%$ & $78 \%$ \\
\hline 146 & 14.3136 & 61.9817 & 20.1714 & 7.3792 & 715.7793 & $30 \%$ & $71 \%$ & $82 \%$ & $23 \%$ & $22 \%$ & $93 \%$ \\
\hline 147 & 14.1336 & 60.2739 & 20.8586 & 7.3792 & 716.2160 & $28 \%$ & $74 \%$ & $93 \%$ & $17 \%$ & $22 \%$ & $92 \%$ \\
\hline 148 & 14.3136 & 60.2350 & 20.6913 & 7.3792 & 716.0953 & $29 \%$ & $71 \%$ & $93 \%$ & $18 \%$ & $22 \%$ & $92 \%$ \\
\hline 149 & 13.8408 & 60.3456 & 20.2310 & 5.7980 & 722.9539 & $51 \%$ & $78 \%$ & $92 \%$ & $23 \%$ & $61 \%$ & $74 \%$ \\
\hline 150 & 14.0252 & 60.1744 & 19.7064 & 7.3792 & 722.6284 & $32 \%$ & $75 \%$ & $93 \%$ & $28 \%$ & $22 \%$ & $75 \%$ \\
\hline 151 & 14.5142 & 60.7141 & 19.7164 & 5.7980 & 719.6693 & $51 \%$ & $68 \%$ & $90 \%$ & $28 \%$ & $61 \%$ & $83 \%$ \\
\hline 152 & 14.8741 & 60.3285 & 19.2865 & 5.7980 & 719.6764 & $52 \%$ & $63 \%$ & $92 \%$ & $32 \%$ & $61 \%$ & $83 \%$ \\
\hline 153 & 14.7817 & 60.5681 & 19.2197 & 5.7980 & 719.5987 & $52 \%$ & $64 \%$ & $91 \%$ & $32 \%$ & $61 \%$ & $83 \%$ \\
\hline 154 & 15.6065 & 60.8887 & 19.5327 & 5.7980 & 718.0555 & $49 \%$ & $53 \%$ & $89 \%$ & $29 \%$ & $61 \%$ & $87 \%$ \\
\hline 155 & 14.3555 & 61.2191 & 20.4670 & 5.7980 & 717.1479 & $49 \%$ & $70 \%$ & $87 \%$ & $21 \%$ & $61 \%$ & $90 \%$ \\
\hline 156 & 14.2207 & 60.9932 & 20.5122 & 5.7980 & 717.0914 & $49 \%$ & $72 \%$ & $88 \%$ & $20 \%$ & $61 \%$ & $90 \%$ \\
\hline 157 & 14.2207 & 60.8284 & 20.9273 & 5.7980 & 717.0842 & $48 \%$ & $72 \%$ & $89 \%$ & $16 \%$ & $61 \%$ & $90 \%$ \\
\hline 158 & 15.6065 & 60.5588 & 18.6509 & 8.2577 & 725.2547 & $25 \%$ & $53 \%$ & $91 \%$ & $38 \%$ & $0 \%$ & $68 \%$ \\
\hline 159 & 15.2207 & 59.8368 & 20.8187 & 8.2577 & 719.4964 & $16 \%$ & $58 \%$ & $95 \%$ & $17 \%$ & $0 \%$ & $83 \%$ \\
\hline 160 & 15.2207 & 59.8044 & 20.7963 & 8.2577 & 719.5635 & $16 \%$ & $58 \%$ & $95 \%$ & $17 \%$ & $0 \%$ & $83 \%$ \\
\hline 161 & 15.0207 & 59.2639 & 19.2798 & 8.2577 & 725.6042 & $23 \%$ & $61 \%$ & $99 \%$ & $32 \%$ & $0 \%$ & $67 \%$ \\
\hline 162 & 15.6065 & 59.4557 & 19.2238 & 7.3792 & 726.0846 & $32 \%$ & $53 \%$ & $98 \%$ & $32 \%$ & $22 \%$ & $66 \%$ \\
\hline 163 & 15.0207 & 59.2967 & 19.5771 & 7.3792 & 725.1592 & $31 \%$ & $61 \%$ & $98 \%$ & $29 \%$ & $22 \%$ & $68 \%$ \\
\hline 164 & 13.2207 & 61.0038 & 20.6531 & 7.3792 & 716.7770 & $30 \%$ & $86 \%$ & $88 \%$ & $19 \%$ & $22 \%$ & $91 \%$ \\
\hline 165 & 13.3555 & 61.1973 & 20.4932 & 5.9736 & 716.9006 & $48 \%$ & $85 \%$ & $87 \%$ & $20 \%$ & $57 \%$ & $90 \%$ \\
\hline 166 & 14.7812 & 62.0419 & 19.4133 & 7.3792 & 719.2174 & $32 \%$ & $64 \%$ & $82 \%$ & $31 \%$ & $22 \%$ & $84 \%$ \\
\hline 167 & 14.5136 & 61.3297 & 19.8860 & 5.7980 & 718.7829 & $51 \%$ & $68 \%$ & $86 \%$ & $26 \%$ & $61 \%$ & $85 \%$ \\
\hline 168 & 14.7812 & 61.2792 & 19.7701 & 5.7980 & 719.0266 & $50 \%$ & $64 \%$ & $86 \%$ & $27 \%$ & $61 \%$ & $85 \%$ \\
\hline 169 & 14.0505 & 60.9606 & 18.6518 & 7.3792 & 725.8881 & $36 \%$ & $75 \%$ & $88 \%$ & $38 \%$ & $22 \%$ & $66 \%$ \\
\hline 170 & 14.4265 & 60.9960 & 18.4767 & 7.3792 & 726.8657 & $36 \%$ & $69 \%$ & $88 \%$ & $39 \%$ & $22 \%$ & $64 \%$ \\
\hline 171 & 14.7812 & 61.8483 & 19.5491 & 7.3792 & 719.0938 & $31 \%$ & $64 \%$ & $83 \%$ & $29 \%$ & $22 \%$ & $84 \%$ \\
\hline 172 & 14.7812 & 61.8159 & 19.4957 & 7.3792 & 719.1609 & $31 \%$ & $64 \%$ & $83 \%$ & $30 \%$ & $22 \%$ & $84 \%$ \\
\hline 173 & 14.5136 & 61.8159 & 19.6197 & 8.2577 & 719.1609 & $23 \%$ & $68 \%$ & $83 \%$ & $29 \%$ & $0 \%$ & $84 \%$ \\
\hline 174 & 13.1181 & 62.4439 & 20.3365 & 7.3792 & 717.2122 & $31 \%$ & $88 \%$ & $79 \%$ & $22 \%$ & $22 \%$ & $89 \%$ \\
\hline 175 & 14.5136 & 62.4492 & 19.8929 & 8.2577 & 717.2666 & $22 \%$ & $68 \%$ & $79 \%$ & $26 \%$ & $0 \%$ & $89 \%$ \\
\hline 176 & 14.5136 & 61.9126 & 19.9932 & 8.2577 & 717.1323 & $21 \%$ & $68 \%$ & $83 \%$ & $25 \%$ & $0 \%$ & $90 \%$ \\
\hline 177 & 14.5136 & 60.4055 & 20.3002 & 7.3792 & 718.6664 & $29 \%$ & $68 \%$ & $92 \%$ & $22 \%$ & $22 \%$ & $86 \%$ \\
\hline 178 & 12.9284 & 61.5858 & 20.5625 & 8.2577 & 716.8914 & $21 \%$ & $91 \%$ & $85 \%$ & $20 \%$ & $0 \%$ & $90 \%$ \\
\hline 179 & 13.1181 & 62.0484 & 20.2701 & 8.2577 & 717.1133 & $22 \%$ & $88 \%$ & $82 \%$ & $22 \%$ & $0 \%$ & $90 \%$ \\
\hline 180 & 14.9413 & 61.2790 & 18.0720 & 5.7980 & 727.6208 & $55 \%$ & $62 \%$ & $86 \%$ & $43 \%$ & $61 \%$ & $62 \%$ \\
\hline 181 & 14.5136 & 61.3615 & 20.0766 & 8.2577 & 718.1755 & $21 \%$ & $68 \%$ & $86 \%$ & $24 \%$ & $0 \%$ & $87 \%$ \\
\hline 182 & 14.5457 & 60.5910 & 18.5135 & 5.7980 & 726.0632 & $55 \%$ & $68 \%$ & $91 \%$ & $39 \%$ & $61 \%$ & $66 \%$ \\
\hline 183 & 14.9413 & 61.3064 & 18.1093 & 5.7980 & 727.3524 & $55 \%$ & $62 \%$ & $86 \%$ & $43 \%$ & $61 \%$ & $63 \%$ \\
\hline 184 & 14.5457 & 60.5910 & 18.4202 & 5.7980 & 726.0632 & $55 \%$ & $68 \%$ & $91 \%$ & $40 \%$ & $61 \%$ & $66 \%$ \\
\hline 185 & 13.4821 & 62.2730 & 21.0476 & 6.8521 & 716.3638 & $34 \%$ & $83 \%$ & $80 \%$ & $15 \%$ & $35 \%$ & $92 \%$ \\
\hline
\end{tabular}


Table A.1. All the acquired Pareto solutions for the smart home appliance scheduling benchmark (continued).

\begin{tabular}{|c|c|c|c|c|c|c|c|c|c|c|c|}
\hline \multirow[b]{2}{*}{$\#$} & \multicolumn{5}{|c|}{ Objectives } & \multicolumn{6}{|c|}{ Utility scores in terms of } \\
\hline & Unsafety & Cost & Delay & PAR & $\begin{array}{c}\mathrm{CO}_{2} \\
\text { emission }\end{array}$ & $\begin{array}{c}\text { Overall } \\
\text { performance }\end{array}$ & Unsafety & Cost & Delay & PAR & $\begin{array}{c}\mathrm{CO}_{2} \\
\text { emission }\end{array}$ \\
\hline 186 & 13.4821 & 62.4377 & 20.4962 & 5.7980 & 716.3709 & $50 \%$ & $83 \%$ & $79 \%$ & $20 \%$ & $61 \%$ & $92 \%$ \\
\hline 187 & 14.5136 & 61.7713 & 20.0256 & 7.3792 & 717.0970 & $30 \%$ & $68 \%$ & $83 \%$ & $25 \%$ & $22 \%$ & $90 \%$ \\
\hline 188 & 14.8065 & 62.6212 & 19.5930 & 8.2577 & 718.8785 & $22 \%$ & $64 \%$ & $78 \%$ & $29 \%$ & $0 \%$ & $85 \%$ \\
\hline 189 & 14.5136 & 61.8182 & 20.3324 & 7.3792 & 718.6522 & $29 \%$ & $68 \%$ & $83 \%$ & $22 \%$ & $22 \%$ & $86 \%$ \\
\hline 190 & 14.8065 & 61.9225 & 19.7583 & 8.2577 & 719.0797 & $22 \%$ & $64 \%$ & $83 \%$ & $27 \%$ & $0 \%$ & $84 \%$ \\
\hline 191 & 14.5136 & 62.0538 & 19.8999 & 8.2577 & 718.4546 & $22 \%$ & $68 \%$ & $82 \%$ & $26 \%$ & $0 \%$ & $86 \%$ \\
\hline 192 & 14.5136 & 60.7659 & 19.5954 & 7.3792 & 722.7390 & $32 \%$ & $68 \%$ & $90 \%$ & $29 \%$ & $22 \%$ & $75 \%$ \\
\hline 193 & 15.0631 & 61.1721 & 18.0313 & 5.7980 & 727.3383 & $55 \%$ & $61 \%$ & $87 \%$ & $44 \%$ & $61 \%$ & $63 \%$ \\
\hline 194 & 15.2136 & 61.3981 & 17.9809 & 5.7980 & 727.3948 & $55 \%$ & $58 \%$ & $86 \%$ & $44 \%$ & $61 \%$ & $62 \%$ \\
\hline 195 & 12.9284 & 62.5168 & 20.0203 & 7.3792 & 718.1382 & $32 \%$ & $91 \%$ & $79 \%$ & $25 \%$ & $22 \%$ & $87 \%$ \\
\hline 196 & 12.8408 & 62.5973 & 20.3016 & 7.3792 & 717.0914 & $32 \%$ & $92 \%$ & $78 \%$ & $22 \%$ & $22 \%$ & $90 \%$ \\
\hline 197 & 14.6786 & 61.2371 & 20.6427 & 7.3792 & 715.4032 & $28 \%$ & $66 \%$ & $87 \%$ & $19 \%$ & $22 \%$ & $94 \%$ \\
\hline 198 & 14.3604 & 61.2371 & 20.6775 & 7.3792 & 715.4032 & $28 \%$ & $70 \%$ & $87 \%$ & $19 \%$ & $22 \%$ & $94 \%$ \\
\hline 199 & 12.7025 & 61.0248 & 20.9104 & 7.3792 & 715.2125 & $30 \%$ & $94 \%$ & $88 \%$ & $16 \%$ & $22 \%$ & $95 \%$ \\
\hline 200 & 14.5812 & 61.2508 & 20.6619 & 7.3792 & 715.2690 & $28 \%$ & $67 \%$ & $87 \%$ & $19 \%$ & $22 \%$ & $95 \%$ \\
\hline 201 & 13.9954 & 60.7175 & 20.9286 & 5.7980 & 715.6962 & $48 \%$ & $76 \%$ & $90 \%$ & $16 \%$ & $61 \%$ & $93 \%$ \\
\hline 202 & 16.9143 & 63.8164 & 16.4717 & 5.7980 & 720.0298 & $57 \%$ & $34 \%$ & $71 \%$ & $59 \%$ & $61 \%$ & $82 \%$ \\
\hline 203 & 17.0170 & 63.7985 & 16.2233 & 5.7980 & 720.5454 & $58 \%$ & $33 \%$ & $71 \%$ & $61 \%$ & $61 \%$ & $81 \%$ \\
\hline 204 & 17.0170 & 63.0336 & 16.2494 & 5.7980 & 722.6644 & $58 \%$ & $33 \%$ & $76 \%$ & $61 \%$ & $61 \%$ & $75 \%$ \\
\hline 205 & 17.0170 & 63.3123 & 16.1666 & 5.7980 & 723.1146 & $58 \%$ & $33 \%$ & $74 \%$ & $61 \%$ & $61 \%$ & $74 \%$ \\
\hline 206 & 16.6350 & 62.9733 & 19.6836 & 5.7980 & 716.7419 & $46 \%$ & $38 \%$ & $76 \%$ & $28 \%$ & $61 \%$ & $91 \%$ \\
\hline 207 & 16.4350 & 63.0007 & 19.6884 & 5.7980 & 716.4735 & $47 \%$ & $41 \%$ & $76 \%$ & $28 \%$ & $61 \%$ & $91 \%$ \\
\hline 208 & 17.4317 & 63.5465 & 17.3139 & 5.7980 & 718.2905 & $53 \%$ & $27 \%$ & $73 \%$ & $51 \%$ & $61 \%$ & $87 \%$ \\
\hline 209 & 17.1099 & 63.4934 & 17.5999 & 5.7980 & 715.8325 & $52 \%$ & $32 \%$ & $73 \%$ & $48 \%$ & $61 \%$ & $93 \%$ \\
\hline 210 & 16.6988 & 62.7554 & 16.0713 & 5.7980 & 727.2426 & $59 \%$ & $38 \%$ & $77 \%$ & $62 \%$ & $61 \%$ & $63 \%$ \\
\hline 211 & 16.6988 & 63.0341 & 15.9150 & 5.7980 & 727.6929 & $60 \%$ & $38 \%$ & $76 \%$ & $64 \%$ & $61 \%$ & $62 \%$ \\
\hline 212 & 17.3099 & 63.6615 & 17.3631 & 5.7980 & 715.9986 & $53 \%$ & $29 \%$ & $72 \%$ & $50 \%$ & $61 \%$ & $93 \%$ \\
\hline 213 & 17.5241 & 63.5670 & 17.4538 & 5.7980 & 718.0009 & $52 \%$ & $26 \%$ & $72 \%$ & $49 \%$ & $61 \%$ & $87 \%$ \\
\hline 214 & 17.0170 & 63.4589 & 17.3143 & 5.7980 & 716.7173 & $53 \%$ & $33 \%$ & $73 \%$ & $51 \%$ & $61 \%$ & $91 \%$ \\
\hline 215 & 16.8488 & 63.5465 & 17.0416 & 5.7980 & 717.1675 & $55 \%$ & $35 \%$ & $73 \%$ & $53 \%$ & $61 \%$ & $90 \%$ \\
\hline 216 & 16.8488 & 63.6916 & 16.9903 & 5.7980 & 716.8232 & $55 \%$ & $35 \%$ & $72 \%$ & $54 \%$ & $61 \%$ & $90 \%$ \\
\hline 217 & 16.4509 & 62.7894 & 19.5575 & 5.7980 & 717.7272 & $47 \%$ & $41 \%$ & $77 \%$ & $29 \%$ & $61 \%$ & $88 \%$ \\
\hline 218 & 16.0367 & 62.8235 & 19.6282 & 5.7980 & 717.3033 & $48 \%$ & $47 \%$ & $77 \%$ & $29 \%$ & $61 \%$ & $89 \%$ \\
\hline 219 & 16.8037 & 63.8872 & 19.5354 & 5.7980 & 715.4207 & $46 \%$ & $36 \%$ & $71 \%$ & $29 \%$ & $61 \%$ & $94 \%$ \\
\hline 220 & 16.6406 & 62.7620 & 19.4548 & 5.7980 & 717.9956 & $47 \%$ & $38 \%$ & $77 \%$ & $30 \%$ & $61 \%$ & $87 \%$ \\
\hline 221 & 16.7872 & 63.0548 & 19.4907 & 5.7980 & 715.9776 & $46 \%$ & $36 \%$ & $76 \%$ & $30 \%$ & $61 \%$ & $93 \%$ \\
\hline 222 & 16.3290 & 63.0908 & 19.5748 & 5.7980 & 716.1252 & $47 \%$ & $43 \%$ & $75 \%$ & $29 \%$ & $61 \%$ & $92 \%$ \\
\hline 223 & 16.0108 & 63.0908 & 19.6325 & 5.7980 & 716.1252 & $48 \%$ & $47 \%$ & $75 \%$ & $28 \%$ & $61 \%$ & $92 \%$ \\
\hline 224 & 15.9026 & 63.2819 & 19.7192 & 5.7980 & 716.1252 & $48 \%$ & $49 \%$ & $74 \%$ & $28 \%$ & $61 \%$ & $92 \%$ \\
\hline 225 & 15.0473 & 63.4531 & 20.0844 & 5.7980 & 715.1278 & $48 \%$ & $61 \%$ & $73 \%$ & $24 \%$ & $61 \%$ & $95 \%$ \\
\hline 226 & 16.7246 & 63.4485 & 16.0845 & 5.7980 & 724.5290 & $59 \%$ & $37 \%$ & $73 \%$ & $62 \%$ & $61 \%$ & $70 \%$ \\
\hline 227 & 16.0170 & 62.8571 & 17.4775 & 5.7980 & 716.9415 & $54 \%$ & $47 \%$ & $77 \%$ & $49 \%$ & $61 \%$ & $90 \%$ \\
\hline 228 & 16.1168 & 63.4053 & 19.8602 & 5.7980 & 715.6859 & $47 \%$ & $46 \%$ & $73 \%$ & $26 \%$ & $61 \%$ & $93 \%$ \\
\hline 229 & 15.6102 & 63.4531 & 19.9504 & 5.7980 & 715.1278 & $48 \%$ & $53 \%$ & $73 \%$ & $25 \%$ & $61 \%$ & $95 \%$ \\
\hline
\end{tabular}


Table A.1. All the acquired Pareto solutions for the smart home appliance scheduling benchmark (continued).

\begin{tabular}{|c|c|c|c|c|c|c|c|c|c|c|c|}
\hline \multirow[b]{2}{*}{ \# } & \multicolumn{5}{|c|}{ Objectives } & \multicolumn{6}{|c|}{ Utility scores in terms of } \\
\hline & Unsafety & Cost & Delay & PAR & $\begin{array}{c}\mathrm{CO}_{2} \\
\text { emission }\end{array}$ & $\begin{array}{c}\text { Overall } \\
\text { performance }\end{array}$ & Unsafety & Cost & Delay & PAR & $\begin{array}{c}\mathrm{CO}_{2} \\
\text { emission }\end{array}$ \\
\hline 230 & 15.4350 & 63.4394 & 19.9664 & 5.7980 & 715.2620 & $48 \%$ & $55 \%$ & $73 \%$ & $25 \%$ & $61 \%$ & $95 \%$ \\
\hline 231 & 16.8488 & 63.3073 & 16.2750 & 5.7980 & 726.9442 & $58 \%$ & $35 \%$ & $74 \%$ & $60 \%$ & $61 \%$ & $64 \%$ \\
\hline 232 & 15.5108 & 63.8872 & 19.6028 & 5.7980 & 715.4207 & $49 \%$ & $54 \%$ & $71 \%$ & $29 \%$ & $61 \%$ & $94 \%$ \\
\hline 233 & 16.8170 & 62.4509 & 17.0874 & 5.7980 & 717.8897 & $55 \%$ & $36 \%$ & $79 \%$ & $53 \%$ & $61 \%$ & $88 \%$ \\
\hline 234 & 16.8170 & 62.8481 & 17.0022 & 5.7980 & 717.4218 & $55 \%$ & $36 \%$ & $77 \%$ & $54 \%$ & $61 \%$ & $89 \%$ \\
\hline 235 & 16.8170 & 62.5599 & 16.9742 & 5.7980 & 717.7043 & $55 \%$ & $36 \%$ & $79 \%$ & $54 \%$ & $61 \%$ & $88 \%$ \\
\hline 236 & 17.1135 & 63.7973 & 18.2044 & 5.7980 & 713.2298 & $50 \%$ & $32 \%$ & $71 \%$ & $42 \%$ & $61 \%$ & $100 \%$ \\
\hline 237 & 17.5241 & 63.7836 & 18.0555 & 5.7980 & 713.3640 & $50 \%$ & $26 \%$ & $71 \%$ & $44 \%$ & $61 \%$ & $100 \%$ \\
\hline 238 & 17.2059 & 63.7836 & 18.1527 & 5.7980 & 713.3640 & $50 \%$ & $30 \%$ & $71 \%$ & $43 \%$ & $61 \%$ & $100 \%$ \\
\hline 239 & 16.7246 & 63.4580 & 16.0320 & 5.7980 & 724.7762 & $59 \%$ & $37 \%$ & $73 \%$ & $63 \%$ & $61 \%$ & $69 \%$ \\
\hline 240 & 17.6215 & 63.5533 & 17.4930 & 5.7980 & 718.1351 & $52 \%$ & $25 \%$ & $73 \%$ & $49 \%$ & $61 \%$ & $87 \%$ \\
\hline 241 & 17.7241 & 63.6313 & 17.4408 & 5.7980 & 716.1046 & $52 \%$ & $23 \%$ & $72 \%$ & $49 \%$ & $61 \%$ & $92 \%$ \\
\hline 242 & 17.3099 & 63.2076 & 17.4845 & 5.7980 & 715.9986 & $53 \%$ & $29 \%$ & $75 \%$ & $49 \%$ & $61 \%$ & $93 \%$ \\
\hline 243 & 15.7184 & 62.8775 & 19.9174 & 5.7980 & 716.7736 & $48 \%$ & $51 \%$ & $77 \%$ & $26 \%$ & $61 \%$ & $91 \%$ \\
\hline 244 & 17.2072 & 63.6505 & 17.9371 & 5.7980 & 713.4983 & $51 \%$ & $30 \%$ & $72 \%$ & $45 \%$ & $61 \%$ & $99 \%$ \\
\hline 245 & 16.8255 & 63.7301 & 18.4221 & 5.7980 & 717.6390 & $50 \%$ & $36 \%$ & $72 \%$ & $40 \%$ & $61 \%$ & $88 \%$ \\
\hline
\end{tabular}

\section{Biographies}

Ali Kaveh was born in 1948 in Tabriz, Iran. After graduation from the Department of Civil Engineering at the University of Tabriz in 1969, he continued his studies on Structures in the Imperial College of Science and Technology at London University and received his MSc and PhD degrees in 1970 and 1974, respectively. He then joined the Iran University of Science and Technology. Professor Kaveh is the author of 620 papers published in international journals and 155 papers presented at national and international conferences. He has authored 23 books in Persian and 10 books in
English published by Wiley, Research Studies Press, American Mechanical Society, and Springer. Professor Kaveh is a fellow of the Iranian Academy of Science, World Academy of Sciences, and European Academy of Sciences and Arts.

Yasin Vazirinia was born in Isfahan, Iran, in 1992. He completed his MSc at Iran University of Science and Technology, Tehran, Iran and is currently a PhD student in the field of Construction Management at the same university. His main research interests are optimization and applications in construction management. 